\title{
STOML2 Interacts with PHB through Activating MAPK Signaling Pathway to Promote Colorectal Cancer Proliferation.
}

\section{Wenhui Ma}

Southern Medical University

\section{Yuehong Chen}

Southern Medical University

Wenyi Li

Southern Medical University

Zhuoluo Xu

Southern Medical University

\section{Zhigang Wei}

Southern Medical University

\section{Tingyu Mou}

Southern Medical University

\section{Zhaokun Wu}

Southern Medical University

\section{Mingzhen Cheng}

Southern Medical University

Yini Zou

Southern Medical University

\section{Yu Zhu}

Southern Medical University

\section{Weijie Zhou}

Southern Medical University

Yan Geng ( $\sim$ gengyan666@163.com )

Southern Medical University

\section{Research}

Keywords: Colorectal Cancer, STOML2, PHB, MAPK signaling pathway, Proliferation

Posted Date: September 8th, 2020

DOl: https://doi.org/10.21203/rs.3.rs-69940/v1 
License: (c) (i) This work is licensed under a Creative Commons Attribution 4.0 International License. Read Full License 


\section{Abstract}

\section{Background}

Overexpression of STOML2 has been widely reported in a variety of cancer, yet few has detailed its function and regulatory mechanism. This study aims to reveal the clinicopathologic significance and oncologic function of STOML2 in colorectal cancer, explore its specific mechanism by means of yeast two-hybrid assay and bioinformatics.

\section{Methods}

Expression level of STOML2 in normal colon and CRC tissue from biobank in Nanfang Hospital was detected by pathologic methods. The malignant proliferation of CRC induced by STOML2 was validated via gain-of-function and loss-of-function experiments, with novel techniques applied, such as organoid culture, orthotopic model and endoscopy monitoring. Yeast two-hybrid assay was conducted to screen interacting proteins of STOML2, followed by bioinformatics to predict biological process and signaling pathway of candidate proteins. Target protein with most functional similarity to STOML2 was validated with co-immunoprecipitation, and immunofluorescence were conducted to co-localize STOML2 and PHB. Pathway regulated by STOML2 was detected with immunoblotting, and subsequent experimental therapy was conducted with RAF inhibitor Sorafenib.

\section{Results}

STOML2 was significantly overexpressed in colorectal cancer and its elevation was associated with unfavorable prognosis. Knockdown of STOML2 suppressed proliferation of colorectal cancer, thus attenuated subcutaneous and orthotopic tumor growth, while overexpressed STOML2 promoted proliferation in cell lines and organoids. A list of 13 interacting proteins was screened out by yeast twohybrid assay. DTYMK and PHB were identified to be most similar to STOML2 according to bioinformatics in terms of biological process and signaling pathways; however, co-immunoprecipitation confirmed interaction between STOML2 and PHB, rather than DTYMK, despite its highest rank in previous analysis. Co-localization between STOML2 and PHB was confirmed in cell lines and tissue level. Furthermore, knockdown of STOML2 downregulated phosphorylation of RAF1, MEK1/2, ERK1/2 and ELK1 on the MAPK signaling pathway, indicating common pathway activated by STOML2 and PHB in colorectal cancer proliferation.

\section{Conclusions}

This study demonstrated that in colorectal cancer, STOML2 expression is elevated and interacts with PHB through activating MAPK signaling pathway, to promote proliferation both in vitro and in vivo. In addition, combination of screening assay and bioinformatics marks great significance in methodology to explore regulatory mechanism of protein of interest. 


\section{Background}

Colorectal cancer (CRC) is the third most prevalent malignant disease (10.2\% of 18.1 million for incidence), and the second leading cause of cancer related death worldwide (9.2\% of 9.6 million for mortality) [1, 2]. The 5-year overall survival rate of patients with CRC is about 56.9-57.6\% [3].Malignant proliferation, invasion-metastasis and chemo-resistance are the main causes of relapse and poor prognosis in CRC, and despite advancing diagnostic techniques and comprehensive therapy, our understanding of underlying mechanism of its malignant phenotype is still limited $[4,5]$. Therefore, it is the primary task for researchers to find out accurate and effective biomarkers, explore how they function in the oncobiologic process of $\mathrm{CRC}$, and assess their prognostic and therapeutic value.

Stomatin-like 2 (aka STOML2, SLP2), which is encoded by gene located on chromosome 9p13, had been initially cloned and identified as a novel member of stomatin family in human erythrocytes, lacking NH2terminal hydrophobic domain [6]. Under physiological condition, STOML2 was identified within inner mitochondria membrane and faces the intermembrane space, interacted with certain component of the inner mitochondria membrane, and served as a regulator of biogenesis and the activity of mitochondria $[7,8]$

Since Zhang et al discovered the elevation of STOML2 in human esophageal squamous cell carcinoma in 2006 [9], researchers in the recent decade have successively discovered STOML2 as an overexpressed biomarker implicated poor prognosis in pan-cancers, such as endometrial adenocarcinoma [10], glioma cells [11], papillary thyroid cancer [12], cervical cancer [13], epithelial ovarian cancer [14, 15], colorectal cancer [16, 17], liver cancer [18], head and neck squamous cell carcinoma [19], etc. Additionally, our research team had previously discovered the elevated expression and association with poor prognosis of STOML2 in gastric cancer [20], and furthermore revealed a positive feedback loop of STOML2 to promote gastric cancer progression [21]. Albeit discovery of STOML2 overexpression in multiple cancers and a handful of tracing to regulatory mechanism were reported, systemic assessment of STMOL2 is still absent. Recent decades have witnessed the thriving technology of high-throughput sequencing, along with a growing body of databases and bioinformatics techniques, presenting more and more effective methods in the exploration of molecular function and regulatory mechanism [22-24].

Herein, the present study focused on the oncological function and regulatory mechanism of STOML2 in $\mathrm{CRC}$. On the basis of STOML2 overexpression in CRC, multiple research models (including cell lines, organoids and animals) were utilized to demonstrate pivotal role of STOML2 in promoting CRC proliferation. Additionally, through yeast two-hybrid assay, thirteen proteins were screened out to be candidates interacting with STOML2. Following bioinformatics analysis of STOML2 and its interaction candidates in two large CRC datasets, downstream signaling pathway of STOML2 was identified, and experimental therapy was conducted.

\section{Methods}




\section{Tissue specimens}

The use of human samples was approved by the Ethics Committee of Nanfang Hospital, Southern Medical University. Human tissue samples were collected from Department of Pathology, Nanfang Hospital, Southern Medical University (Guangzhou, China). All patients given informed consents. None of these patients received chemotherapy or radiotherapy before operation.

\section{Cell culture}

Human CRC cell lines (HCT116, HT29, SW480, SW620) and murine CRC cell lines (MC38, CT26) were purchased from Cell Resource Center, Shanghai Institute of Biochemistry and Cell Biology at the Chinese Academy of Sciences (Shanghai, China) and maintained at the Department of Pathology, Southern Medical University (Guangzhou, China). Human cell lines were cultured in RPMI-1640 medium, and murine cell lines were cultured in DMEM medium containing $10 \%$ fetal bovine serum (Gibco), at $37^{\circ} \mathrm{C}$, in the atmosphere of $5 \% \mathrm{CO}_{2}$.

\section{Immunohistochemistry and immunofluorescent staining}

For immunohistochemistry $(\mathrm{IHC})$ staining, tissue slides were deparaffinized and hydrated, incubated with antibody anti-STOML2 (1:1000, Proteintech, Wuhan, China) and anti-Ki-67 (1:200, BD, CA, US) overnight at $4{ }^{\circ} \mathrm{C}$. For negative controls, the antibodies were replaced with normal non-immune serum. Tissue slides were reviewed and scored by two independent observers, based on percentage of positive cells and the degree of positive staining. Positive cells at each intensity of staining were recorded on a scale of 0-3 (0, no staining; 1 , weak staining = light yellow; 2 , moderate staining = yellowish brown; 3 , strong staining = brown). A score of $\geq 2$ with at least $50 \%$ of malignant cells with positive STOML2 staining was classified as tumors with high expression of STOML2, and $<50 \%$ of malignant cells with nuclear staining or $<2$ intensity score was classified as tumors with low expression of STOML2. For cell immunofluorescent (IF) staining, a multiplexed tyramide signal amplification method (TSA; PerkinElmer, Inc., US) was performed on 4- $\mu \mathrm{m}$ sections for detection of the co-localization of STOML2 and PHB proteins. Prior to each immunofluorescence labeling, antigens were retrieved with a single microwave step. Each labeling cycle consists of application of a primary antibody, a secondary antibody conjugated to horse radish peroxidase (HRP), and TSA conjugated to a fluorophore. Tissue slides were incubated with antibody against STOML2 and PHB for 30 min respectively. TSA conjugated Fluorescein was used for STOML2 and CY 5 for PHB. Images were captured using inverted confocal microscope (Olympus, Japan) and suite software. Images were processed using Image $\mathrm{J}$ and Photoshop CS5 software (Adobe Systems Inc., San Jose, CA).

\section{Establishment of stably transfected cell lines}


SW620 cells were infected with STOML2-knockdown (shSTOML2) or scramble shRNA (Scr; GENECHEM, Shanghai, China). SW480 cells were infected with STOML2-overexpressed (3×Flag-STOML2) or control lentivirus (mock; GENECHEM, Shanghai, China). MC38 and CT26 cells were infected with STOML2knockdown (shSTOML2) or control lentivirus (Scr; GENECHEM, Shanghai, China). Cells were seeded in 6well plates at a density of $2 \times 10^{\wedge} 5$ cells per well, $24 \mathrm{~h}$ before transfection. $2 \times 10^{\wedge} 6 \mathrm{TU}$ of corresponding lentivirus and $5 \mu \mathrm{g}$ of polybrene (Merck, Darmstadt, Germany) were mixed to $1 \mathrm{~mL}$ serum-free medium to transfect cells. After transfection for $48 \mathrm{~h}, 1 \mu \mathrm{g} / \mathrm{mL}$ of puromycin (Merck, Germany) was added to each well to screen out the stably transfected cells, and the cells were then transferred to conventional medium. Transfection efficiency was confirmed by quantitative reverse transcription polymerase chain reaction (qRT-PCR) and immunoblot assay.

\section{Total RNA extraction and real-time quantitative PCR}

Total RNA was extracted with Trizol reagent (TaKaRa, Dalian China) following manufacturer's protocol. cDNA synthesis was performed with PrimeScript ${ }^{\mathrm{TM}} \mathrm{RT}$ reagent Kit (TaKaRa, Dalian China). qRT-PCR was carried out using SYBR Premix Ex Taq ${ }^{\text {TM }}$ II (TaKaRa, Dalian China) on ABI-7500 instrument (Applied BioSystems). Data were normalized to the mean Ct values of housekeeping gene GAPDH and calculated using $-\Delta \Delta \mathrm{Ct}$ method to compare variation in gene expression.

\section{Cell proliferation, colony formation assay and transwell assay}

For Cell Counting Kit-8 (CCK8) assay, cells were seeded in 96-well plates at a density of 800 or 5000 cells per well, respectively for murine or human CRC cell lines, one day before proliferation assay. Where indicated, SW480 cells were pre-treated with $10 \mu \mathrm{M}$ sorafenib or equal volume of DMSO before CCK8 assay. Cell medium of each well was discarded and replaced by CCK-8 reagent $2 \mathrm{~h}$ before testing. Absorbance value was detected at $450 \mathrm{~nm}$ wavelength by Microplate Reader (Perkin Elmer, MA, US), continuously for 4 days. For colony formation assay, cells were sufficiently distributed in 6-well plates with $3 \mathrm{ml}$ complete medium. After 14 -day incubation at $37^{\circ} \mathrm{C}$ in an atmosphere of $5 \% \mathrm{CO} 2$, the colonies formed by single cells were fixed in $75 \%$ ethanol and stained with Giemsa for quantification. For transwell assay, $2 \times 10^{\wedge} 5$ cells were seeded with $500 \mu \mathrm{l}$ serum-free medium into transwell chamber (Corning, NY, US), which was insert to 24 well plate. Medium containing $30 \%$ FBS was added to the bottom chamber. After $24 \mathrm{~h}$ incubation, cells invaded into lower chamber were fixed in methanol, stained in crystal violet (Sigma, MO, US) and counted under microscope. All experiments were replicated for three times.

\section{Immunoblot and co-immunoprecipitation assay}

Total protein of cultured cells were extracted using lysis buffer (KeyGEN, Jiangsu, China), with PMSF, protease and phosphatase inhibitor reagents added according to the manufacturer's instruction. Equal 
mass of protein extract was separated in sodium dodecyl sulfatepolyacrylamide gel electrophoresis (SDS-PAGE) and then transferred to PVDF membranes (Merck Millipore, MA, US). After BSA blocking for 1 hour, the protein-loading membranes were incubated with primary antibody overnight at $4^{\circ} \mathrm{C}$. These membranes were then incubated with HRP-conjugated goat anti-mouse or anti-rabbit secondary antibodies (zsbio, Beijing, China) for 1 hour at room temperature. Blotting images were captured and analyzed using Image Lab Software (Bio-Rad, CA, US). GAPDH was set as endogenous reference.

For immunoprecipitation assay, total protein of cultured cells were lysed and incubated with $50 \mu$ proteinA Sepharose beads (Santa Cruz Biotechnology, TX, US), and anti-His, anti-PHB or anti-SLP2 antibody as where indicated, at $4{ }^{\circ} \mathrm{C}$ overnight with gentle mixing and anti-lgG was set as a control. Then the samples were washed and denaturized for western blotting.

Referred antibodies were purchased from commercial sources as follows: anti-STOML2 (1:1000, Proteintech, Wuhan, China), anti-GAPDH (1:1000, Proteintech, Wuhan, China), anti-PHB (1:500, Genetex, CA, US), anti-His (1:800, Proteintech, Wuhan, China), anti-IgG (Santa Cruz Biotechnology, TX, US), antiRAF1 (1:100, Cell Signaling Technology, MA, US), anti-p-RAF1 (1:100, Cell Signaling Technology, MA, US), anti-MEK1/2 (1:200, Cell Signaling Technology, MA, US), anti-p-MEK1/2 (1:100, Cell Signaling Technology, MA, US), anti-ERK1/2 (1:500, Cell Signaling Technology, MA, US), anti-p-ERK1/2 (1:200, Cell Signaling Technology, MA, US).

\section{Isolation, culture, transfection and immunofluorescent staining ofprimary murine colon tumors}

Colon tumors of 16-week $A p c^{\mathrm{Min} /+}$ mice were isolated, collected and embedded onto Matrigel (BD Biosciences) at $50 \mu \mathrm{l}$ per well in 24-well plates, following previously published methods [25]. The culture medium was DMEM/F12, supplemented with 1 unit/ml of penicillin, $1 \mu \mathrm{g} / \mathrm{ml}$ of streptomycin, $2.5 \mathrm{ng} / \mathrm{ml}$ of amphotericin B, $10 \mathrm{mmol} / \mathrm{L}$ HEPES, 2mM Glutamax, 1× N2 supplement, 1× B27 supplement, and 50 $\mathrm{ng} / \mathrm{ml}$ murine EGF. Culture medium was changed every 2 days and organoids were passaged by mechanical disruption once a week. Organoid transfection was conducted as described protocol [26]. Briefly, organoids were trypsinized for $10 \mathrm{~min}$ at $37^{\circ} \mathrm{C}$, to obtain single cell suspension. $2 \times 10^{\wedge} 6 \mathrm{TU}$ of corresponding lentivirus and $5 \mu \mathrm{g}$ of polybrene (Merck, Germany) were mixed to $1 \mathrm{~mL}$ serum-free medium to transfect cells. After incubated for $48 \mathrm{~h}, 1 \mu \mathrm{g} / \mathrm{mL}$ of puromycin (Merck, Germany) was added to each well to screen out the stably transfected cells, which were re-embedded to Matrigel afterwards. For IF staining [27], organoids were grown in matrigel plated onto an 8-well chamber slide (Lab-Tek II, 154534). After culture medium was discarded, organoids were fixed in 4\% PFA-PME (50 mM PIPES, $2.5 \mathrm{mM} \mathrm{MgCl}{ }_{2}$, $5 \mathrm{mM}$ EDTA) for 20 minutes, then permeabilized in $0.5 \%$ Triton for 20 minutes and blocked in IF Buffer (PBS, $0.2 \%$ Triton, $0.05 \%$ Tween, $1 \% \mathrm{BSA}$ ) for $1 \mathrm{~h}$. Organoids immunofluorescent (IF) staining was conducted as previously described. Chamber slide were incubated with antibody against E-cadherin and Ki67 for 30 min. Images were captured using inverted confocal microscope (Olympus, Japan) and suite 
software. Images were processed using Image $\mathrm{J}$ and Photoshop CS5 software (Adobe Systems Inc., San Jose, CA).

\section{Animals and tumor growth assay}

All mice experiments were approved by Animal Research Ethics Committee of Southern Medical University and proceeded in accordance with the guidelines on the care and use of animals for scientific purposes. Stable-transfected murine CRC cells $\left(2 \times 10^{6}\right)$ of MC38-Scr, MC38-shSTOML2 or CT26-Scr, CT26shSTOML2 suspended in $200 \mu \mathrm{l}$ PBS were injected subcutaneously into the left or right hind limb of 6week-old male wild type (WT) C57BL/ 6 or BALB/c mice ( $n=7 /$ group), accordingly. Tumor size was measured every three days with a vernier caliper, and tumor volume was calculated as $0.52 \times \mathrm{L} \times \mathrm{W}^{2}\left(\mathrm{~cm}^{3} ; \mathrm{L}\right.$ stands for length and $\mathrm{W}$ for width of the tumor). Mice were anesthetized and sacrificed at indicated time, around 4 weeks after injection. Tumors were dissected, measured and photographed, then fixed with formalin and embedded in paraffin for further immnuohistological assessment.

\section{Orthotopic model and murine endoscopy monitoring}

Murine CRC cell lines MC38 and CT26 were suspended at density of $2 \times 10^{\wedge} 6$ in $50 \mu \mathrm{L}$ PBS for each injection. Mice were anesthetized by inhaling 1.5 to $2 \%$ isoflurane (RWD Life Science Co., Ltd, Shenzhen, China). Optical colonoscopy was performed using a Karl Storz (Tuttlingen, Germany) Image 1 HD Camera System, Image 1 HUB CCU, 175 Watt Xenon Light Source, and Richard Wolf 1.9mm/9.5 Fr Integrated Telescope (part number 8626.431). Indicated cells were injected into mouse colonic lamina propria under colonoscopy, at around $1 \mathrm{~cm}$ from anal orifice, using a custom injection needle (Hamilton Inc., 33-gauge, small Hub RN NDL, 6 inches long, point 4, 45 degree bevel, like part number 7803-05), syringe (Hamilton Inc. part number 7656-01), and transfer needle (Hamilton Inc. part number 7770-02). Tumor growth was assessed by serial endoscopy monitoring and recording carried out every 6 days after injection, and tumor size was scored by the diameter of the colonic lumen occupied by tumor [28]. Mice were anesthetized and sacrificed at indicated time, around 30 days after injection. Total colons were dissected, measured and photographed, then fixed in formalin and embedded in paraffin for further immnuohistological assessment.

\section{Yeast two-hybrid screening assay}

Human CRC cell line SW620 was selected to construct CRC CDNA library. Following total RNA extraction, cDNA single/double strand synthesis and purification, SW620 cDNA library was co-transformed with pGADT7-Rec (Clontech) into Saccharomyces cerevisiae strain Y187. Transformation efficiency and titer of CRC cDNA library was verified. The full-length of STOML2 coding sequence was cloned to inframe with the Gal4 DNA binding domain of the bait plasmid pGBKT7 (Clontech) by PCR. pGBKT7-STOML2 plasmid was transformed into Saccharomyces cerevisiae strain $\mathrm{Y} 2 \mathrm{H}$ to establish bait strain, and double 
strand library cDNA synthesized from SW620 was co-transfected with pGADT7-Rec plasmid into Y187 to establish library host strain. Bait strain was first tested to be non-toxic to Y2H, and pGBKT7-STOML2 could not activate reporter gene by itself. Bait and library host strain were then co-cultured at $30 \mathrm{C}$ with 30 $50 \mathrm{rpm}$ swirling, and after mating for 20 hours, zygotes in typical three-lobed shape were present in the mating culture. Mating mixture was spread on three $100 \mathrm{~mm}$ media (SD/-Trp, SD/-Leu, SD/-Leu/-Trp) at dilution of $1: 10,1: 100,1: 10^{\wedge} 3,1: 10^{\wedge} 4$. After 3 to 5 days, the number of colonies screened and mating efficiency were calculated, and remaining mixture was spread on forty-four $150 \mathrm{~mm}$ QDO/A media (SD/Ade/-His/-Leu/-Trp/Aba) for 3-to-5-days culture. On QDO/A media, clones over $2 \mathrm{~mm}$ at diameter were selected and transferred to QDO/X/A media, to underwent higher selective pressure. Blue-stained colonies on QDO/X/A media were randomly selected to verify multiple prey genes by amplification with polymerase chain reaction. These plasmids with prey genes (pGADT7-Prey) were extracted from each blue-stained monoclone, and respectively co-transfected with pGBKT7-STOML2 and pGBKT7 into competent yeast strain pGBKT7-S+pGADT7-Prey and pGBKT7+pGADT7-Prey. To retest the interaction and exclude false positive in yeast, these two $\mathrm{Y} 2 \mathrm{H}$ strain were spread on DDO/X and QDO/X/A conditioned media, with pGBKT7-53+pGADT7-T set as positive control and pGBKT7-lam+pGADT7-T as negative control.

\section{Statistical analysis}

Data were all presented as mean \pm standard error of mean (SEM) unless otherwise annotated. Statistical analysis was performed by two-tail unpaired Student's $t$ test for experiments where two means were compared. Two-way analysis of variance (ANOVA) was used to compare means of three or more experimental groups. Factorial design ANOVA was used to analyze experiments with two independent variables. Gene set enrichment analyses (GSEA) were conducted using GSEA 4.0.3 (Broad Institute, MA, US) $[29,30]$. Statistical analyses were performed using GraphPad Prism software 5.0 (GraphPad Software, CA, US) and SPSS software (Version 22.0, IL, US).

\section{Results}

\section{STOML2 is highly expressed and predicts poor prognosis in CRC patients.}

Following discovery of overexpression of STOML2 in gastric cancer and investigation into specific molecular mechanism of this gene afterwards, we were intrigued whether STOML2 presented analogous high-expressed profile and biological effect in CRC. Therefore, 215 CRC patients were randomly selected from bio-bank in General Surgery, Nanfang Hospital, as internal validation of STOML2 expression in tissue level. With clinical samples collected and examined, immunochemistry of patients' resected tissue showed relatively high STOML2 expression in tumor and metastatic lymph nodes, in comparison with normal tissue (Fig. 1A). In tumor-adjacent region, distinction of STOML2 expression between tumor and normal tissue was more visible, a legible margin could be drawn (Fig. 1B). Immuno-blotting assay in 
twelve samples and paired tissues also confirmed higher STOML2 protein expression in tumor (Fig. 1D). Based on STOML2 expression level in tissue, these patients were then divided into high expression group and low expression group. Statistical analyses showed significant difference in tumor clinicopathological characteristics, such as T stage, lymph node metastasis, distant metastasis, AJCC classification, between two groups (Table 1). Notably, 10-year follow-up witnessed unfavorable prognosis in survival analysis for those who detected high STOML2 expression in resected tissue between two groups (Fig. 1C; log-rank $p<$ 0.001). Analyses of five public datasets from Gene Expression Omnibus (GEO) in various cancer types demonstrated common elevated expression profile of STOML2, especially in CRC and related metastatic lesions in liver and lung (Fig. 1E-I). Data in BioGPS [31] also supported significant difference in STOML2 expression between normal colon and CRC (Supplementary Fig. 1). 
Table 1

Correlations between SLP2 expression and clinicopathological parameters of CRC patients.

SLP2 expression

$p$ value ${ }^{a}$

Variables

Sex

Male

Female

Age

$<61$

$\geq 61$

Differentiation

Well

Moderate

Poor and undifferentiated

T stage

$\mathrm{T} 1+\mathrm{T} 2$

$\mathrm{T} 3+\mathrm{T} 4$

Lymph node metastasis

Absent (NO)

Present (N1-2)

Distant metastasis

Absent (M0)

Present (M1)

AJCC classification

I

All cases

$(n=215)$

132

83

103

112

81

105

29

30

185

114

101

$62(52.0)$

24 (20.9)

18 (55.0)

68 (29.9)

37 (36.2)

$9(28.1)$

0.026

0.085

65 (64.4)

64 (67.2)

48 (32.8)

40 (37.0)

41 (63.0)

68 (63.8)

20 (71.9)

0.405

0.569

77 (66.2)

52 (65.1)

$\begin{array}{ll}38(35.6) & 65(64.4) \\ 48(32.8) & 64(67.2)\end{array}$

(

$62(52.0)$
$24(20.9)$

168

23

60(38.2)

2 (6.7)

21 (93.3)

12 (45.0)

117 (70.1) 


\begin{tabular}{|c|c|c|c|c|}
\hline & \multirow[b]{2}{*}{$\begin{array}{l}\text { All cases } \\
(n=215)\end{array}$} & \multicolumn{2}{|c|}{ SLP2 expression } & \multirow[t]{2}{*}{$p$ value $^{a}$} \\
\hline Variables & & $\begin{array}{l}\text { Low(\%) } \\
(n=86)\end{array}$ & $\begin{array}{l}\text { High (\%) } \\
(n=129)\end{array}$ & \\
\hline IV & 23 & 2 & 21 & \\
\hline
\end{tabular}

These results suggested that, STOML2 was overexpressed in CRC, correlated with unfavorable clinicopathological characteristics and poor prognosis, which hinted us that it contributed to malignant phenotypes of CRC and was worth further study.

\section{STOML2 promotes CRC cell proliferation and colony formation in vitro.}

To discover the association between STOML2 expression and malignant phenotype of CRC, STOML2 protein level was detected in seven human-derived CRC cell lines, among which SW620 had the highest STOML2 expression and SW480 the lowest (Fig. 2A). Therefore, we established STOML2-knockdown SW620 cell line with lentivirus transfection (shSTOML2), and STOML2-overexpressed SW480 cell line with 3xFlag-tagged STOML2 lentivirus, of which transfection efficiency was confirmed by immunoblotting assay (Fig. 2B \& I). Inhibited cell growth rate (Fig. 2C), less colony formation (Fig. 2E-F), with inhibited migration and invasion abilities (Fig. 2G-H) was observed in STOML2-knockdown groups, compared with negative control. In STOML2-overexpressed SW480, cell proliferation rate was significantly enhanced (Fig. 2J), more colonies were formed (Fig. 2K-L), migration and invasion abilities were significantly enhanced (Fig. 2M-N). These results showed that overexpressed STOML2 significantly enhanced the proliferative ability of human CRC.

\section{STOML2 promotes CRC growth and progression in vivo}

Colon tumors were dissected from 16-week old $\mathrm{ApC}^{\mathrm{Min} /+}$ mice and tumor cells were isolated for 3D culture. Tumor organoids were transfected with STOML2 overexpressing lenti-virus, which yielded substantially larger organoids compared to negative control (Fig. 3A-B). Immunofluorescent staining for epithelial marker E-cadherin and Ki-67 using transfected organoids indicated significantly higher expression of Ki-67 in STOML2-overexpressed epithelial tumor cell (Fig. 3C). These results demonstrated that STOML2 could accelerate proliferation of CRC.

To investigate the biological function of STOML2 in vivo, STOML2-knockdown murine CRC cell strains, MC38-Scr/shSTOML2 and CT26-Scr/shSTOML2, were selected referred to CCK8 results, and injected subcutaneously to mice strains C57-B6 and Balb/c, respectively ( $n=7$ for each group). Tumor size were measured and growth curves were drawn, which showed that tumor in MC38- shSTOML2 and CT26- 
shSTOML2 groups grew more slowly and smaller in size than those in corresponding negative control (Fig. 3D-F, Supplementary Fig. 2A-C). IHC confirmed less staining of proliferation marker Ki67, suggesting knockdown of STOML2 markedly attenuated CRC cell proliferation in vivo (Fig. 3G\&H, Supplementary Fig. 2D-F).

Orthotopic model simulating human colorectal cancer formation was established by implantation of above mentioned MC38/CT26-scr and -shSTOML2 cells into colon mucosa of corresponding mice, via injection under inspection of endoscopy (Fig. 3I). Similar results of slower and smaller tumor growth in shSTOML2 groups compared with negative control (Fig. 3J). Moreover, mice implanted with shSTOML2 cells had significant longer survival than those with control group cells (Fig. $3 \mathrm{~K}$; log-rank $p=0.016$ ). Thus, results above had proven that STOML2 knockdown attenuated proliferation of CRC cells in vivo, both in subcutaneous and orthotopic tumor model.

\section{Yeast two-hybrid assay explored potential targets of STOML2}

To further explore proteins that potentially interacts with STOML2, we next conducted yeast two-hybrid assay. Bait strain PGBKT7-STOML2 was first tested to be non-toxic and could not activate reporter gene by itself (Fig. 4A). Bait and library host strain were then mated for 20 hours, zygotes in typical three-lobed shape were present in the mating culture (Fig. 4B). Mating mixture was spread on three media (SD/-Trp,

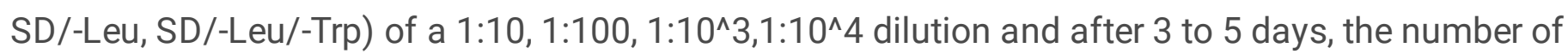
colonies screened was $8.15 \times 10^{6} \mathrm{cfu}$ and mating efficiency was $3.26 \%$. Remaining mixture was then spread on $150 \mathrm{~mm}$ QDO/A media (SD/-Ade/-His/-Leu/-Trp/Aba) for 3 to 5 days culture (Fig. 4C), and clones over $2 \mathrm{~mm}$ at diameter were selected and transferred to QDO/X/A media to underwent higher selective pressure. As a result, all colonies of pGBKT7-STOML2 + pGADT7-T yeasts and positive control grew well on conditioned media and were stained blue (Fig. 4D), which indicated that proteins expressed by pGADT7-T screened from library strain might have possible interaction with STOML2 expressed by bait pGBKT7-STOML2. These plasmids with prey genes (pGADT7-Prey) were extracted from each bluestained monoclone, and respectively co-transfected with pGBKT7-STOML2 and pGBKT7 into yeast (pGBKT7-S + pGADT7-Prey and pGBKT7 + pGADT7-Prey). To retest the interaction and exclude false positive in yeast, these two yeast strains were spread on DDO/X and QDO/X/A conditioned media. The result showed that positive control group grew normally and stained blue on DDO/X and QDO/X/A plates, while the negative control group did not, which guaranteed the reliability of this screening system. pGBKT7-STOML2 + pGADT7-prey group grew well on DDO/X and QDO/X/A plates, while pGBKT7 + pGADT7-Prey group did not grow on QDO/X/A plate and were not stained blue on DDO/X plate, which indicated that the activation of the four reporter genes (-his, -ade, aba and X-a-Gal) was activated by the interaction between STOML2 and prey protein, rather than self-activated by prey. Last but not least, pGBKT7-STOML2 + pGADT7-Prey was stained blue on both DDO/X and QDO/X/A plate, confirmed that these screened prey proteins interacted with STOML2 protein (Fig. 4E). Eventually, sequence of these screened plasmid were determined and a list of 13 prey proteins was identified, among which proteins like "PHB", "PHB2", "FLI1", "DTYMK", “GLP1R” were the most referred (Fig. 4F). 


\section{GSEA analyses revealed DTYMK and PHB were functionally similar with STOML2}

To further validate protein that most possibly interacts with STOML2, we performed GSEA of STOML2 and 13 candidates, using CRC sample expression profiling datasets GSE14333 $(n=290)$ [32] and GSE17538 ( $n=238)$ [33-36] on GEO database (Fig. 5 and Supplementary Fig. $3-5)$. We picked top 20 pathway terms with highest normalized enrichment score (NES) of each gene; as is shown, overexpressed STOML2 was correlated with upregulation in KEGG pathways like "RNA polymerase", "pyrimidine metabolism", "cell cycle", "DNA replication”, indicating its functional connection with proliferation in CRC (Fig. 5A). In these top 20 terms, ranking of prey genes that overlapped with STOML2 was: DTYMK (16 out of 20), PHB (14), PRSS2 (9), PHB2 (8), ATP5B (6), CD8a (2), TCF7L2 (2), GLP1 (1), GLP1R (1). Terms such as "cell cycle", "pyrimidine metabolism", "RNA polymerase", "DNA replication", "mismatch repair", "homologous recombination", were shared between DTYMK/PHB and STOML2 in Kyoto Encyclopedia of Genes and Genomes (KEGG) pathways (Fig. 5 and Supplementary Fig. 3), whereas terms like "ribosome biogenesis", "rRNA metabolic process", "ribonucleoprotein complex subunit organization", "ribonucleoprotein complex biogenesis", "translational elongation" were shared in Gene Ontology (GO) biological process (Supplementary Fig. 4A-C \& 5A-C), which hinted that DTYMK and PHB were the most functionally similar proteins with STOML2 (Fig. 5A-C).

\section{STOML2 interacts with PHB to modulate RAF/MEK/ERK MAPK pathway}

However, binding between STOML2 and DTYMK could not be verified by co-IP (Supplementary Fig. 3F). Since DTYMK was excluded, we turned our focus to PHB. Co-IP confirmed binding interaction of STOML2 and PHB in vitro (Fig. 6A). IF staining in SW620 cell line indicated co-localization of STOML2 and PHB in cytoplasm (Fig. 6B). In comparison of tissue sections from our biobank, IF staining demonstrated low STOML2 and PHB expression in normal colon, and evident co-location between STOML2 and PHB in CRC lesions, besides clear margin at the invasive front in tumor-adjacent tissue (Fig. 6C). STRING database [37] also suggested PHB as functional partner of STOML2 with high score (0.916; Fig. 6D), which indeed had been identified to play indispensable role in the activation of the RAF-MEK-ERK pathway by RAS [38]. In STOML2-knockdown cells, PHB protein expression decreased simultaneously, accompanied by decreased phosphorylation of MAPK pathway proteins, such as RAF, MEK and ERK (Fig. 6E). Experimental therapy targeting MAPK pathway was conducted in STOML2-overexpressed SW480, where cells were treated with RAF1 inhibitor Sorafenib and showed significant attenuation in proliferation assay (Fig. 6F). Results above pointed out that STOML2 interacted with PHB, and potentially promoted CRC proliferation through regulating MAPK pathway and disruption STOML2-PHB interaction or inhibition of MAPK signaling pathway might be therapeutic strategies for STOML2 highly expressed CRC.

\section{Discussion}


Cancer cell is characterized by its ability of infinite proliferation, under influence of persistent activation or inhibition of signaling pathways, triggered by alteration of expression level of certain gene(s) effecting these pathways $[39,40]$. In the present study, STOML2 is the key oncogene screened out from medical center biobank by our research team, which is highly expressed, and related to tumorigenesis, progression and prognosis of gastroenteric cancers.

From precedent researches, we have revealed the elevation of STOML2 in gastric cancer on tissue and molecular level, and its correlation with various clinical indicators, which also verified elevation of STOML2 as an independent prognostic factor for gastric cancer, provided first and valid evidence to explore the specific molecular mechanism of it in malignant proliferation of gastric cancer [21]. In this study, an increase in STOML2 expression in CRC was discovered and its significant association with adverse clinical factors of CRC patients was confirmed, which indicated that STOML2-upregulated CRC exhibits malignant proliferating phenotype such as proliferation, cell cycle, invasion and metastasis.

The biological role of STOML2 is to regulate mitochondrial membrane stability and function; under mitochondrial stress, it interacts with prohibitins, molecular chaperones in respiratory chain complexes [7]. Hu et al. reported that STOML2 activated MEK/ERK signaling and suppressed mitochondrial apoptosis pathway in HeLa cervical cancer cells, through altering the ability of mitochondria to buffer $\mathrm{Ca}^{2+}$ and shape cytosolic $\mathrm{Ca}^{2+}$ signal [41]. Recent related studies also implied that, STOML2 modulated tumor malignancy via IL6-Stat3 pathway in head and neck squamous cell carcinoma [19]. In study conducted by Zhu et al., silence of STOML2 represses migration and invasion ability of liver cancer via inhibiting the NF-KB Pathway [42]. Zhou et al. detected the variations of canonical Wnt/ $\beta$-catenin signaling pathway after SLP-2 inhibition in CRC cells to enhance cell growth [43]. Based on the results of current study and evidence from other researchers, we suppose that similar effect occurred in STOML2elevated CRC cells, through which promotes cancer cell proliferation, and is worth further evaluation. These accumulating evidence indicates that STOML2 broadly participated in various signaling network, and served as an important pro-tumorigenic gene in CRC.

In this study, novel techniques were utilized in the process of exploring STOML2's malignant phenotype, which provided more convincing evidence. Organoid culture along with subsequent IHC staining provides near-physiologic cellular composition and behavior, which permits study of morphology during tumor formation. Orthotopic model combined with routine endoscopic monitoring provides several advantages over traditional subcutaneous xenograft. Orthotopic injection furnishes better simulation of microenvironment for CRC carcinogenesis, while mouse endoscopy is conducive to continuously monitoring tumor burden in vivo without causing damage, and reduces the need for multiple cohorts of experimental mice, in terms of animal welfare.

The pivotal role of STOML2 in CRC progression was validated through current research. For protein overexpressed and linked with malignancy in various cancers, multiple perspectives could be targeted in terms of therapeutic strategy, eg. antibody or recombinant protein treatment for membrane proteins [44, 45], and small molecule drugs targeting downstream pathway of intracellular proteins. In this study,

Page 15/31 
based on subcellular localization of STOML2 in cytoplasm, yeast two-hybrid assay combining bioinformatics analysis was implemented to screen out putative interacting proteins of STOML2, which share similar biological function and signaling pathways. Among 13 putative candidates, we started from DTYMK and PHB with the highest rank, co-IP confirmed interaction between STOML2 and PHB, with significant co-localization in cell line and tissue, but not DTYMK. Other candidates from $\mathrm{Y} 2 \mathrm{H}$ assay also showed promising research value. For instance, since the major pool of STOML2 is associated with mitochondria, ATP synthases like ATP5B and ATP5H also share resemblance with STOML2 in function and subcellular localization; it was reported that STOML2 is an important player in T cell activation by ensuring sustained TCR signaling, which could be connected with CD8A [46-48]. After downstream pathway was identified by further analysis, in vitro treatment was conducted with sorafenib to inhibit STOML2-regulated MAPK pathway, which presented significant attenuation of tumor growth. Furthermore, we shall proceed to in-depth investigation on binding site and interacting domain between STOML2 and PHB, in order to be more pertinent in treatment study.

\section{Conclusions}

In conclusion, this study presents that STOML2 is significantly upregulated in CRC and promotes cancer cell proliferation and tumor growth through MAPK signaling pathway by interaction with PHB, which suggests STOML2 as novel, potent biomarker and therapeutic target in CRC, urging for further investigation.

\section{Abbreviations}

CRC, colorectal cancer; STOML2, stomatin-like 2; CCK8, cell counting kit 8; PMSF, phenylmethylsulfonyl fluoride; PBS, phosphate Buffered Saline; SDS-PAGE, sodium dodecyl sulfatepolyacrylamide gel electrophoresis; PVDF, Poly (vinylidene fluoride); FFPE, formalin fixed paraffin-embedded; HRP, Horseradish Peroxidase; SD, synthetic dropout medium; DDO, double dropout supplement; QDO, quadruple dropout supplement; SEM, standard error of mean; GSEA, gene set enrichment analysis; GEO, gene expression omnibus; PHB, prohibitin; DTYMK, deoxythymidylate kinase; MAPK, mitogen-activated protein kinase.

\section{Declarations}

\section{Ethics approval and consent to participate}

All the patients were informed of sample collection and usage. The tissue samples were collected and used in accordance with approval by the Institutional Ethical Committee Board (Nanfang Hospital, Guangzhou, China). Use of animal was approved by the Nanfang hospital animal ethic committee.

\section{Consent for publication}


All authors consented to publication of the results presented in this manuscript.

\section{Availability of data and materials}

The datasets supporting the conclusions of this article, GSE14333 and GSE17538 are available in Gene Expression Omnibus (GEO) database, "https://www.ncbi.nlm.nih.gov/geo/query/acc.cgi?acc=GSE14333" [32] and "https://www.ncbi.nIm.nih.gov/geo/query/acc.cgi?acc=GSE17538" [33, 34, 36].

\section{Competing interests}

All authors declared no conflict of interests.

\section{Funding}

This work was supported by the National Natural Science Foundation of China $(81672446,81902970)$, Natural Science Foundation of Guangdong Province (No. 2017A030310115 and No. 2019A1515011325), State's Key Project of Research and Development Plan (2017YFC0108300), Guangdong Provincial Natural Science Foundation for Distinguished Young Scientists (2015A030306048), Guangzhou science and technology collaborative innovation major projects (201704020071), Southern Medical University Clinical Research Start-Up Project (LC2016ZD003) and Key Clinical Specialty Discipline Construction Program ((2012)121).

\section{Authors' contributions}

YG and WZ conceived research design, supervised data and drafted the manuscript. WM, YC and WL were mainly responsible for conducting experiments, acquisition of data and analysis. WM, ZX carried out western blot analysis and organoid-related experiments. YC, ZW performed the animal experiments and bioinformatics, statistical analysis. WL, MC, YZ performed IHC staining and functional experiments. ZW, TM collected clinical samples and data for this study. All authors read and approved the final manuscript.

\section{Acknowledgements}

We would like to express gratitude to Prof. Guoxin Li and Department of General Surgery, Nanfang Hospital for providing CRC specimens, also towards Central Laboratory of Southern Medical University and its staffs for technical support.

\section{References}


1. Bray F, Ferlay J, Soerjomataram I, Siegel RL, Torre LA, Jemal A. Global cancer statistics 2018 : GLOBOCAN estimates of incidence and mortality worldwide for 36 cancers in 185 countries. CA Cancer J Clin. 2018;68(6):394-424.

2. Torre LA, Bray F, Siegel RL, Ferlay J, Lortet-Tieulent J, Jemal A. Global cancer statistics, 2012. CA Cancer J Clin. 2015;65(2):87-108.

3. Allemani C, Matsuda T, Di Carlo V, Harewood R, Matz M, Nikšić M, et al. Global surveillance of trends in cancer survival 2000-14 (CONCORD-3): analysis of individual records for $37+\square 513+\llbracket 025$ patients diagnosed with one of 18 cancers from 322 population-based registries in 71 countries. The Lancet. 2018;391(10125):1023-75.

4. Keum N, Giovannucci E. Global burden of colorectal cancer: emerging trends, risk factors and prevention strategies. Nat Rev Gastroenterol Hepatol. 2019;16(12):713-32.

5. Dekker E, Tanis PJ, Vleugels JLA, Kasi PM, Wallace MB. Colorectal cancer. The Lancet. 2019;394(10207):1467-80.

6. Wang Y, Morrow JS. Identification and characterization of human SLP-2, a novel homologue of stomatin (band 7.2b) present in erythrocytes and other tissues. J Biol Chem. 2000;275(11):8062-71.

7. Wang Y, Cao W, Yu Z, Liu Z. Downregulation of a mitochondria associated protein SLP-2 inhibits tumor cell motility, proliferation and enhances cell sensitivity to chemotherapeutic reagents. Cancer Biol Ther. 2009;8(17):1651-8.

8. Hajek P, Chomyn A, Attardi G. Identification of a novel mitochondrial complex containing mitofusin 2 and stomatin-like protein 2. J Biol Chem. 2007;282(8):5670-81.

9. Zhang L, Ding F, Cao W, Liu Z, Liu W, Yu Z, et al. Stomatin-like protein 2 is overexpressed in cancer and involved in regulating cell growth and cell adhesion in human esophageal squamous cell carcinoma. Clin Cancer Res. 2006;12(5):1639-46.

10. Cui Z, Zhang L, Hua Z, Cao W, Feng W, Liu Z. Stomatin-like protein 2 is overexpressed and related to cell growth in human endometrial adenocarcinoma. Oncol Rep. 2007;17(4):829-33.

11. Song L, Liu L, Wu Z, Lin C, Dai T, Yu C, et al. Knockdown of stomatin-like protein 2 (STOML2) reduces the invasive ability of glioma cells through inhibition of the NF-kappaB/MMP-9 pathway. J Pathol. 2012;226(3):534-43.

12. Liu Z, Yang Y, Zhang Y, Ye X, Wang L, Xu G. Stomatin-like protein 2 is associated with the clinicopathological features of human papillary thyroid cancer and is regulated by TGF-beta in thyroid cancer cells. Oncol Rep. 2014;31(1):153-60.

13. Xiao B, Xie Z, Guo L, Wu J, Zhang H. Stomatin-like protein 2 expression is associated with clinical survival in patients with cervical cancer. Int J Clin Exp Pathol. 2015;8(2):1804-9.

14. Sun F, Ding W, He JH, Wang XJ, Ma ZB, Li YF. Stomatin-like protein 2 is overexpressed in epithelial ovarian cancer and predicts poor patient survival. BMC Cancer. 2015;15:746.

15. Guo XY, Guo HF, Guo HM. Clinical significance of SLP-2 in epithelial ovarian cancer and its regulatory effect on the Notch signaling pathway. Eur Rev Med Pharmacol Sci. 2020;24(4):1666-71. 
16. Zhang L, Liu F. Expression of SLP-2 gene and CCBE1 are associated with prognosis of rectal cancer. Eur Rev Med Pharmacol Sci. 2017;21(6):1214-8.

17. Liu Q, Li A, Wang L, He W, Zhao L, Wu C, et al. Stomatin-like Protein 2 Promotes Tumor Cell Survival by Activating the JAK2-STAT3-PIM1 Pathway, Suggesting a Novel Therapy in CRC. Mol Ther Oncolytics. 2020;17:169-79.

18. Zhu W, Li W, Geng Q, Wang X, Sun W, Jiang H, et al. Silence of Stomatin-Like Protein 2 Represses Migration and Invasion Ability of Human Liver Cancer Cells via Inhibiting the Nuclear Factor Kappa B (NF-kappaB) Pathway. Med Sci Monit. 2018;24:7625-32.

19. Qu H, Jiang W, Wang Y, Chen P. STOML2 as a novel prognostic biomarker modulates cell proliferation, motility and chemo-sensitivity via IL6-Stat3 pathway in head and neck squamous cell carcinoma. Am J Transl Res. 2019;11(2):683-95.

20. Liu D, Zhang L, Shen Z, Tan F, Hu Y, Yu J, et al. Increased levels of SLP-2 correlate with poor prognosis in gastric cancer. Gastric cancer. 2013;16(4):498-504.

21. Ma W, Xu Z, Wang Y, Li W, Wei Z, Chen T, et al. A Positive Feedback Loop of SLP2 Activates MAPK Signaling Pathway to Promote Gastric Cancer Progression. Theranostics. 2018;8(20):5744-57.

22. Cancer Genome Atlas N. Comprehensive molecular characterization of human colon and rectal cancer. Nature. 2012;487(7407):330-7.

23. Zhou M, Zhao H, Wang Z, Cheng L, Yang L, Shi H, et al. Identification and validation of potential prognostic IncRNA biomarkers for predicting survival in patients with multiple myeloma. J Exp Clin Cancer Res. 2015;34:102.

24. Wang Q, Gan H, Chen C, Sun Y, Chen J, Xu M, et al. Identification and validation of a 44-gene expression signature for the classification of renal cell carcinomas. J Exp Clin Cancer Res. 2017;36(1):176.

25. Xue X, Shah YM. In vitro organoid culture of primary mouse colon tumors. J Vis Exp. 2013(75):e50210.

26. Schwank G, Koo BK, Sasselli V, Dekkers JF, Heo I, Demircan T, et al. Functional repair of CFTR by CRISPR/Cas9 in intestinal stem cell organoids of cystic fibrosis patients. Cell Stem Cell. 2013;13(6):653-8.

27. Dow LE, O'Rourke KP, Simon J, Tschaharganeh DF, van Es JH, Clevers H, et al. Apc Restoration Promotes Cellular Differentiation and Reestablishes Crypt Homeostasis in Colorectal Cancer. Cell. 2015;161(7):1539-52.

28. Ernst M, Preaudet A, Putoczki T. Non-invasive assessment of the efficacy of new therapeutics for intestinal pathologies using serial endoscopic imaging of live mice. J Vis Exp. 2015(97).

29. Subramanian A, Tamayo P, Mootha VK, Mukherjee S, Ebert BL, Gillette MA, et al. Gene set enrichment analysis: a knowledge-based approach for interpreting genome-wide expression profiles. Proc Natl Acad Sci U S A. 2005;102(43):15545-50.

30. Mootha VK, Lindgren CM, Eriksson KF, Subramanian A, Sihag S, Lehar J, et al. PGC-1alpharesponsive genes involved in oxidative phosphorylation are coordinately downregulated in human 
diabetes. Nat Genet. 2003;34(3):267-73.

31. Wu C, Orozco C, Boyer J, Leglise M, Goodale J, Batalov S, et al. BioGPS: an extensible and customizable portal for querying and organizing gene annotation resources. Genome Biol. 2009;10(11):R130.

32. Jorissen RN, Gibbs P, Christie M, Prakash S, Lipton L, Desai J, et al. Metastasis-Associated Gene Expression Changes Predict Poor Outcomes in Patients with Dukes Stage B and C Colorectal Cancer. Clin Cancer Res. 2009;15(24):7642-51.

33. Smith JJ, Deane NG, Wu F, Merchant NB, Zhang B, Jiang A, et al. Experimentally derived metastasis gene expression profile predicts recurrence and death in patients with colon cancer. Gastroenterology. 2010;138(3):958-68.

34. Freeman TJ, Smith JJ, Chen X, Washington MK, Roland JT, Means AL, et al. Smad4-mediated signaling inhibits intestinal neoplasia by inhibiting expression of beta-catenin. Gastroenterology. 2012;142(3):562-71. e2.

35. Chen MS, Lo YH, Chen X, Williams CS, Donnelly JM, Criss ZK 2. Growth Factor-Independent 1 Is a Tumor Suppressor Gene in Colorectal Cancer. Mol Cancer Res. 2019;17(3):697-708. nd, et al.

36. Williams CS, Bernard JK, Demory Beckler M, Almohazey D, Washington MK, Smith JJ, et al. ERBB4 is over-expressed in human colon cancer and enhances cellular transformation. Carcinogenesis. 2015;36(7):710-8.

37. Szklarczyk D, Gable AL, Lyon D, Junge A, Wyder S, Huerta-Cepas J, et al. STRING v11: protein-protein association networks with increased coverage, supporting functional discovery in genome-wide experimental datasets. Nucleic Acids Res. 2019;47(D1):D607-D13.

38. Rajalingam K, Wunder C, Brinkmann V, Churin Y, Hekman M, Sievers C, et al. Prohibitin is required for Ras-induced Raf-MEK-ERK activation and epithelial cell migration. Nat Cell Biol. 2005;7(8):837-43.

39. Wan PTC, Garnett MJ, Roe SM, Lee S, Niculescu-Duvaz D, Good VM, et al. Mechanism of Activation of the RAF-ERK Signaling Pathway by Oncogenic Mutations of B-RAF. Cell. 2004;116(6):855-67.

40. Barker N, Clevers H. Mining the Wnt pathway for cancer therapeutics. Nat Rev Drug Discov. 2006;5(12):997-1014.

41. Hu G, Zhang J, Xu F, Deng H, Zhang W, Kang S, et al. Stomatin-like protein 2 inhibits cisplatin-induced apoptosis through MEK/ERK signaling and the mitochondrial apoptosis pathway in cervical cancer cells. Cancer Sci. 2018;109(5):1357-68.

42. Zhu W, Li W, Geng Q, Wang X, Sun W, Jiang H, et al. Silence of Stomatin-Like Protein 2 Represses Migration and Invasion Ability of Human Liver Cancer Cells via Inhibiting the Nuclear Factor Kappa B (NF-kB) Pathway. Med Sci Monit. 2018;24:7625-32.

43. Zhou C, Li Y, Wang G, Niu W, Zhang J, Wang G, et al. Enhanced SLP-2 promotes invasion and metastasis by regulating Wnt/beta-catenin signal pathway in colorectal cancer and predicts poor prognosis. Pathol Res Pract. 2019;215(1):57-67.

44. Davenport AP, Scully CCG, de Graaf C, Brown AJH, Maguire JJ. Advances in therapeutic peptides targeting G protein-coupled receptors. Nat Rev Drug Discov. 2020;19(6):389-413. 
45. Rotte A. Combination of CTLA-4 and PD-1 blockers for treatment of cancer. J Exp Clin Cancer Res. 2019;38(1):255.

46. Christie DA, Kirchhof MG, Vardhana S, Dustin ML, Madrenas J. Mitochondrial and plasma membrane pools of stomatin-like protein 2 coalesce at the immunological synapse during $T$ cell activation. PLoS One. 2012;7(5):e37144.

47. Kirchhof MG, Chau LA, Lemke CD, Vardhana S, Darlington PJ, Marquez ME, et al. Modulation of T cell activation by stomatin-like protein 2 . J Immunol. 2008;181(3):1927-36.

48. Christie DA, Mitsopoulos P, Blagih J, Dunn SD, St-Pierre J, Jones RG, et al. Stomatin-like protein 2 deficiency in $T$ cells is associated with altered mitochondrial respiration and defective CD $4+T$ cell responses. J Immunol. 2012;189(9):4349-60.

\section{Figures}


A
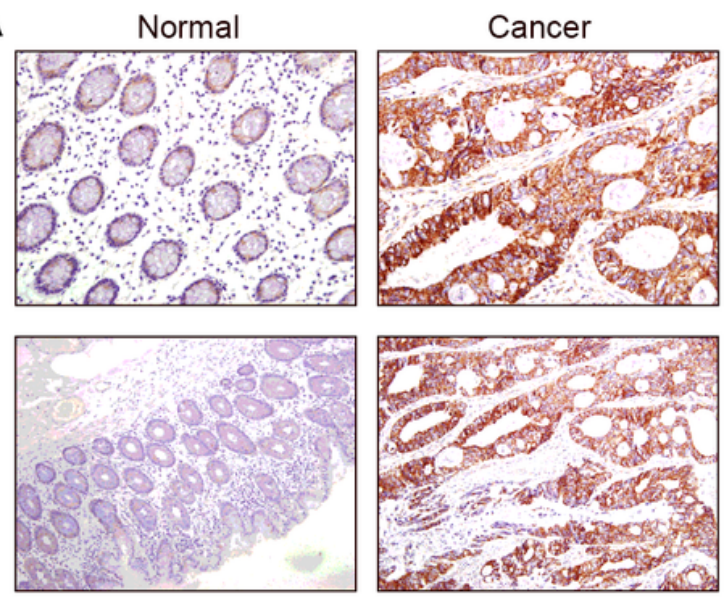

Lymph node
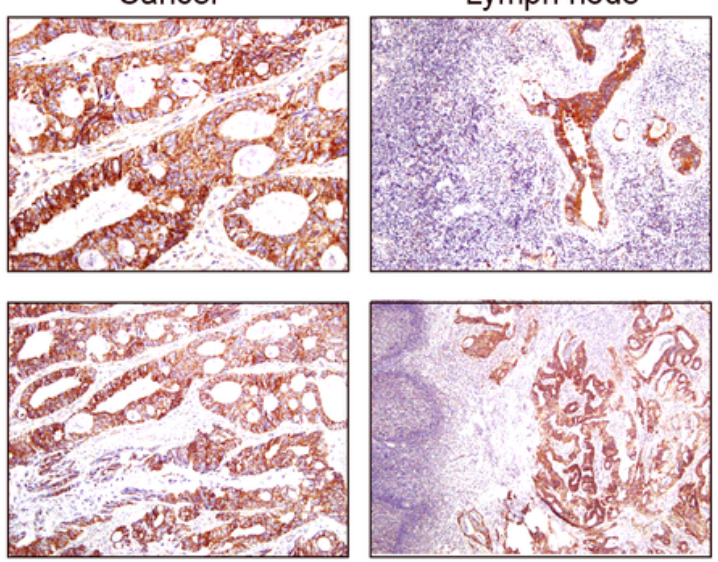

B
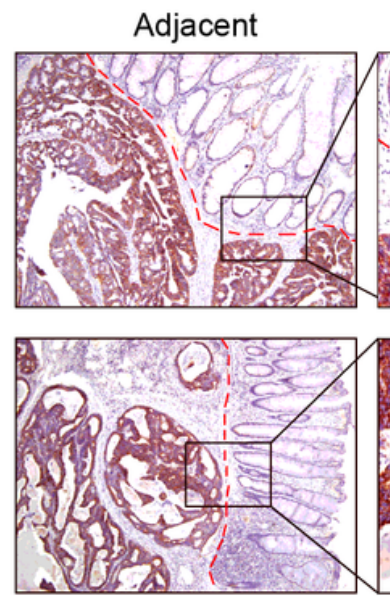

Adjacent
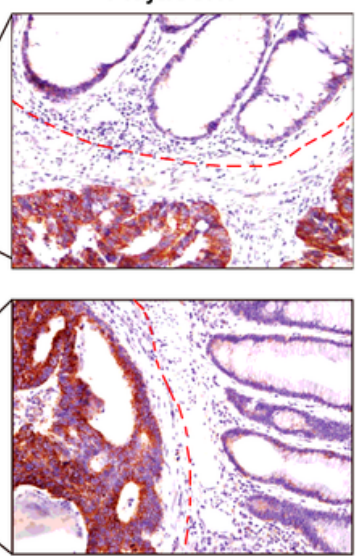

C

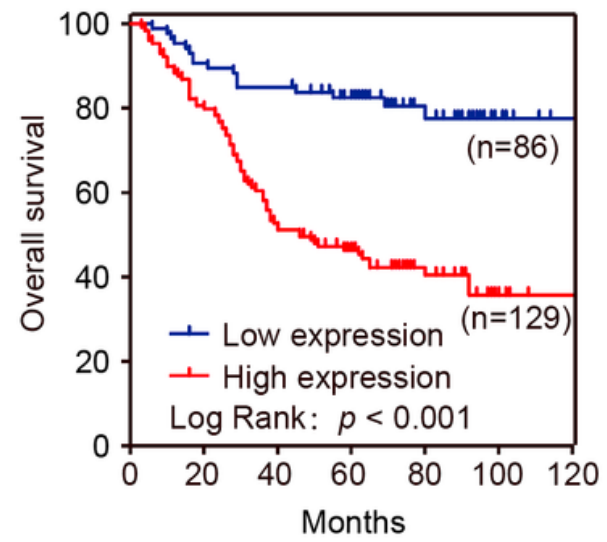

D Case 1 $\frac{\text { Case 2 }}{\mathrm{N} T} \frac{\text { Case 3 }}{\mathrm{N} T} \frac{\text { Case 4 }}{\mathrm{N} T}$

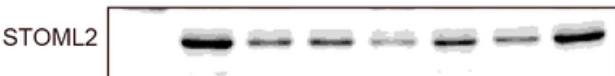

GAPDH

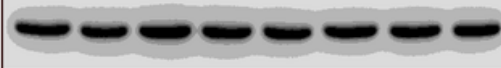

STOML

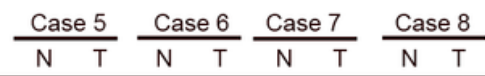

GAPDH
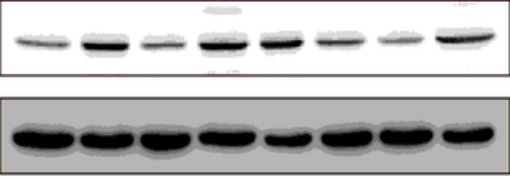

$\mathrm{E}$
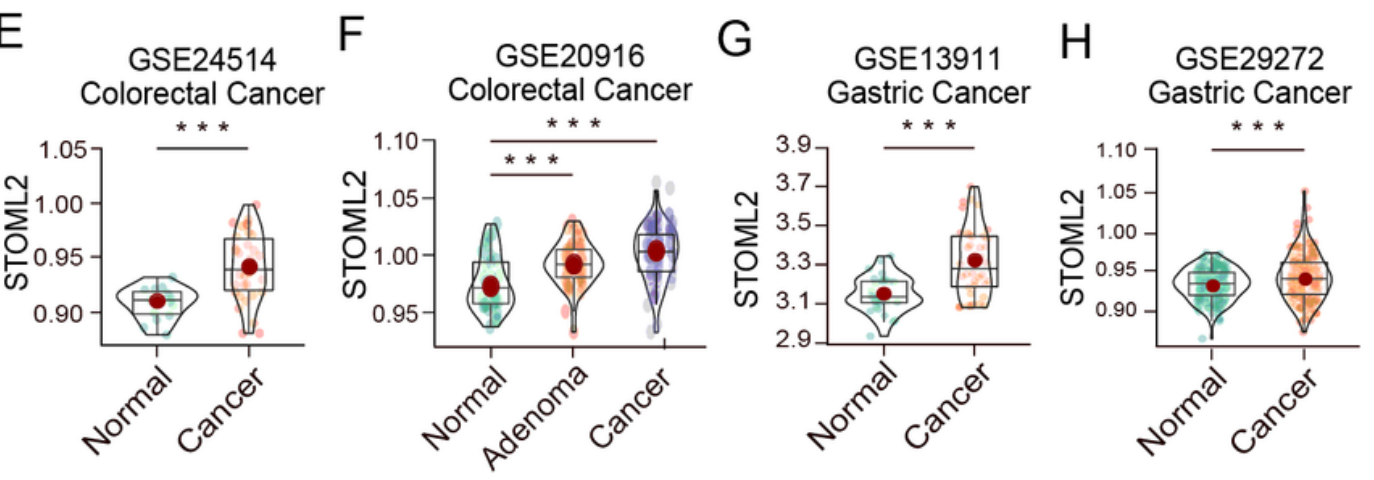

I
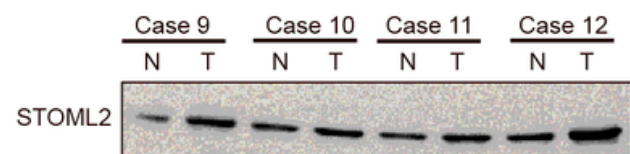

GAPDH
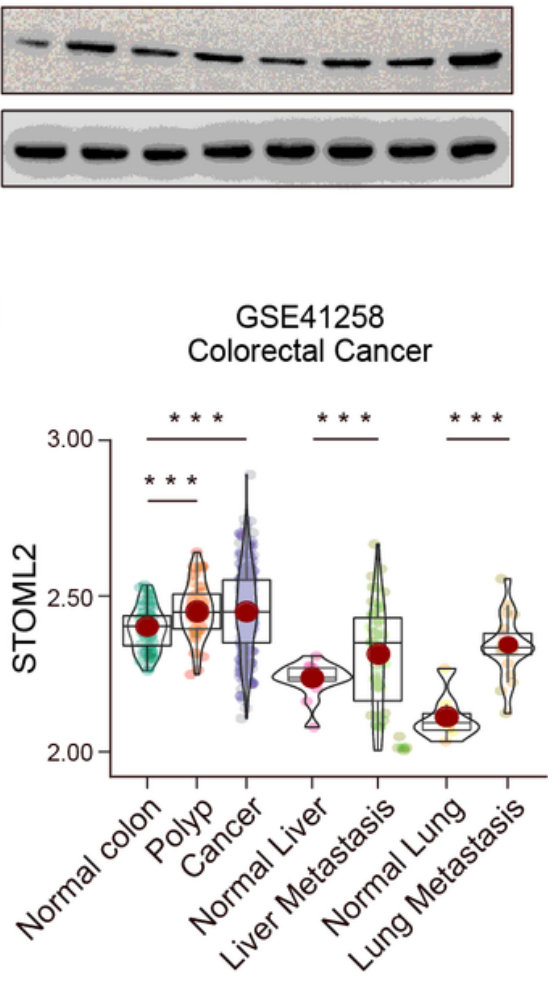

Figure 1

STOML2 is highly expressed and predicts poor prognosis in CRC patients. a Representative images of STOML2 staining in CRC, paired normal, cancer and metastatic lymph nodes tissues of internal cohort. b Representative images of STOML2 staining in CRC tumor adjacent tissues of internal cohort; right: 40x magnified image of box on the left; dotted line outlines invasive front. c Kaplan-Meier curves of CRC patients overall survival with high or low STOML2 expression level of internal cohort. $p \otimes 0.001$, log-rank test. d Immunoblot of STOML2 and GAPDH in normal and tumor tissue of twelve CRC cases from internal cohort; N, normal colon; T, tumor. e-i External validation of STOML2 expression in different cancers from GEO database. (e) GSE24514 dataset, CRC, normal tissue vs. cancer ( $n=15$ vs. 34). (f) GSE20916 dataset, CRC, normal tissue vs. adenoma vs. cancer ( $n=34$ vs. 54 vs. 66). (g) GSE13911 
dataset, GC, normal tissue vs. cancer ( $n=31$ vs. 38). (h) GSE29272 dataset, GC, normal tissue vs. cancer ( $n=134$ vs. 134). (i) GSE41258 dataset, CRC, normal colon polyp vs. cancer ( $n=54$ vs. 49 vs. 186), normal liver vs. liver metastasis ( $n=13$ vs. 47 ), normal lung vs. lung metastasis ( $n=7$ vs. 20). Data was presented as means \pm SEM from five independent experiments. $p$ values were determined using $t$ test. *** $p<0.001$.

A
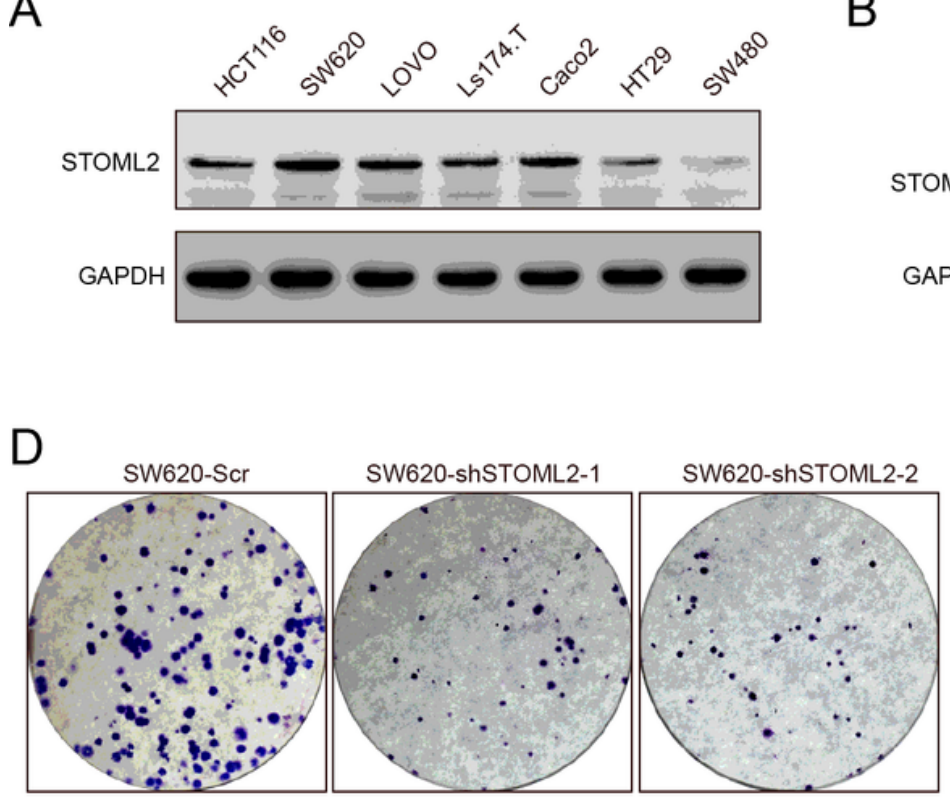

$\mathrm{F}$

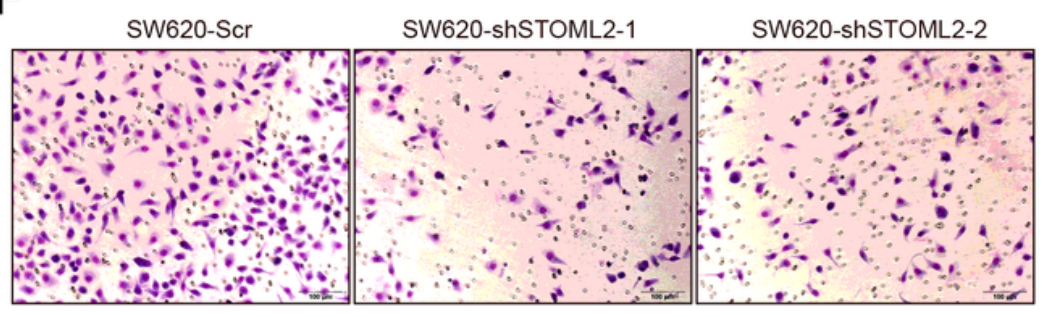

J

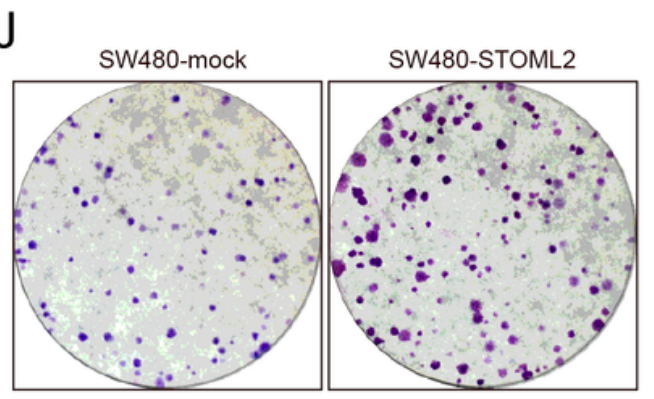

B

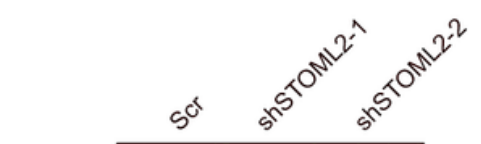

STOML2

GAPDH

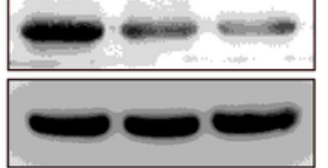

SW620

E
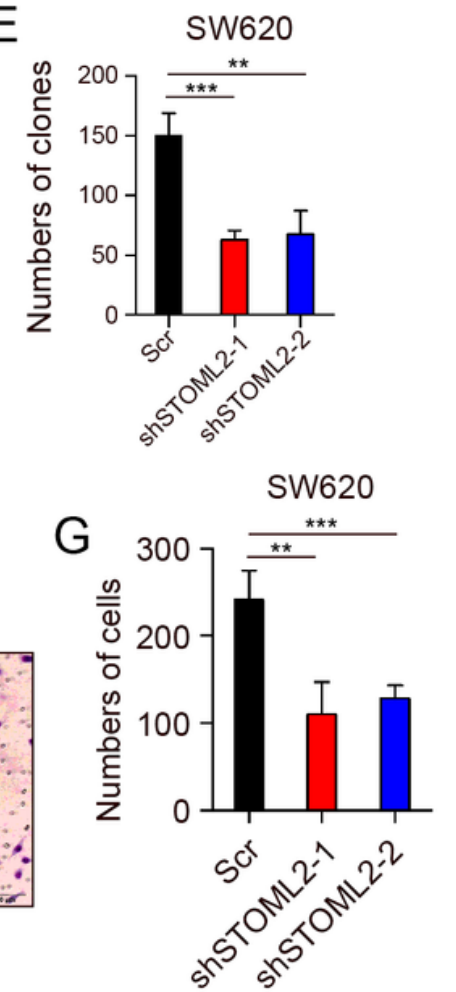

K

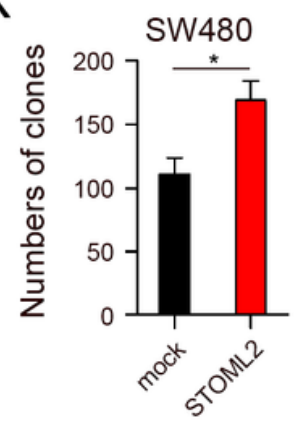

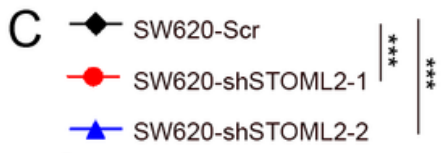

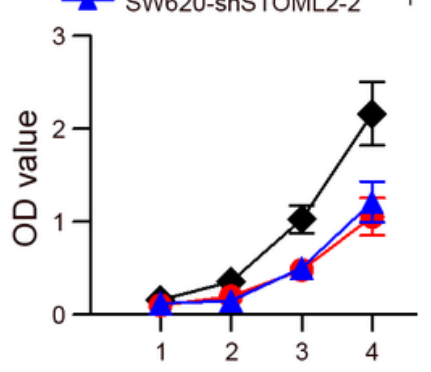

Times (Day)

$\mathrm{H}$
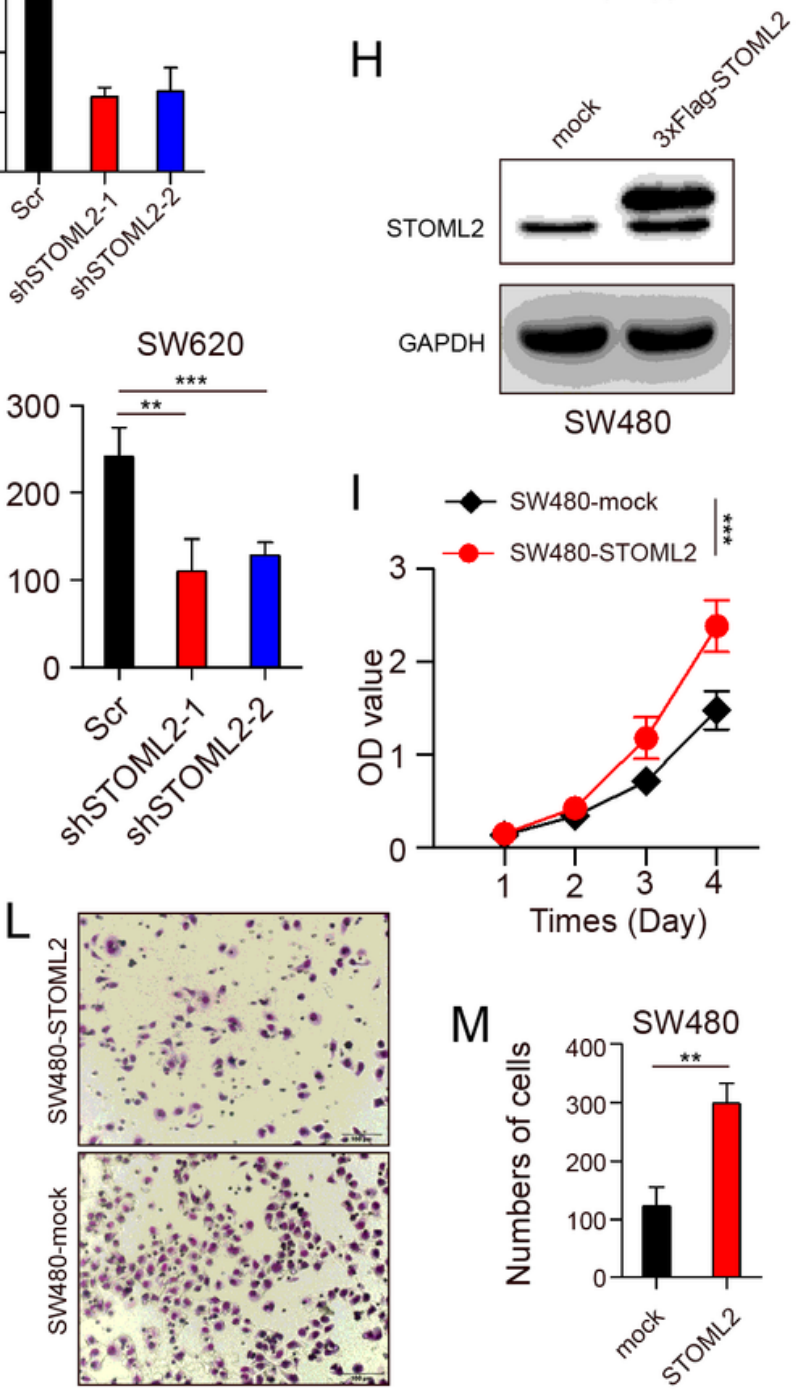

M

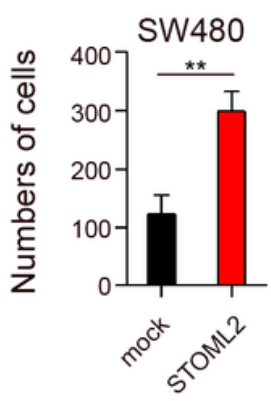

Figure 2 
STOML2 promotes CRC cell proliferation and colony formation in vitro. a Immunoblot of STOML2 and GAPDH in seven CRC cell lines. b Immunoblot of STOML2 and GAPDH in SW620 transfected with STOML2-knockdown lentivirus. c 5000 indicated cells were seeded in 96 wells culture-plates and OD values were measured in CCK8 assay. Data are presented as means \pm SEM from five independent experiments. ${ }^{* *} \mathrm{p}<0.001$, two-way ANOVA. d-e Colony formation of STOML2-knockdown SW620. (d) 5000 indicated cells were seeded in 6 wells culture-plates. Giemsa staining after 14 days of culture. (e) number of colonies formed on each plate. Data presented as means \pm SEM from three independent experiments. ${ }^{* \star} \mathrm{p}<0.001,{ }^{\star \star} \mathrm{p} \otimes 0.01$, one-way ANOVA. f-g Transwell assay of STOML2-knockdown SW620. (f) Representative figures of fixed cells in lower chamber. 5000 indicated cells were suspended in upper chamber. (g) Number of cells counted on each plate. Data presented as means \pm SEM from five independent experiments. ${ }^{\star * *} \mathrm{p}<0.001,{ }^{\star *} \mathrm{p} \otimes 0.01$, one-way ANOVA. $\mathrm{h}$ Immunoblot assay of indicated protein in SW480 transfected with mock and STOML2-overexpressed lentivirus. i 5000 indicated cells were seeded in 96 wells culture-plates and OD values were measured in CCK8 assay. Data was presented as means \pm SEM from five independent experiments. ${ }^{* \star} p<<0.001$, two-way ANOVA. j-k Colony formation of STOML2-overexpressed SW480. 5000 indicated cells were seeded in 6 wells culture-plates. Number of colonies formed on each plate. Data presented as means \pm SEM from five independent experiments. ${ }^{*} p<$ 0.05 , $t$ test. m-n Transwell assay of STOML2-overexpressed SW480. (g) representative figures of fixed cells in lower chamber. 5000 indicated cells were suspended in upper chamber. $(\mathrm{h})$ number of cells

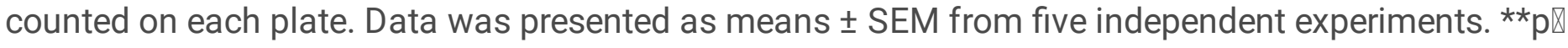
$0.01, \mathrm{t}$ test. 
A

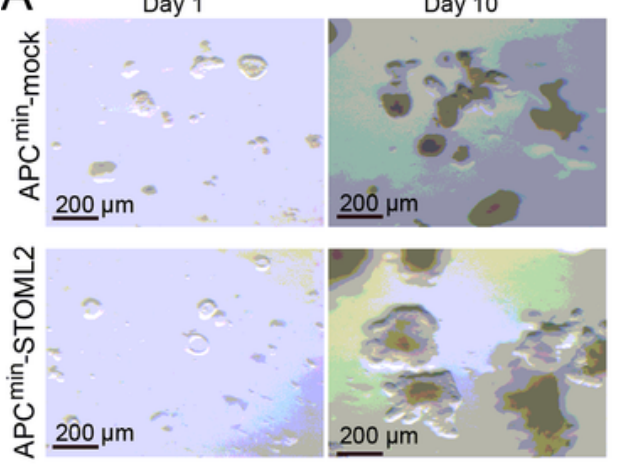

C

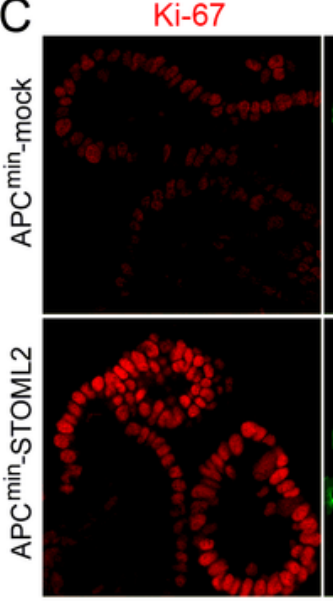

$\mathrm{F}$
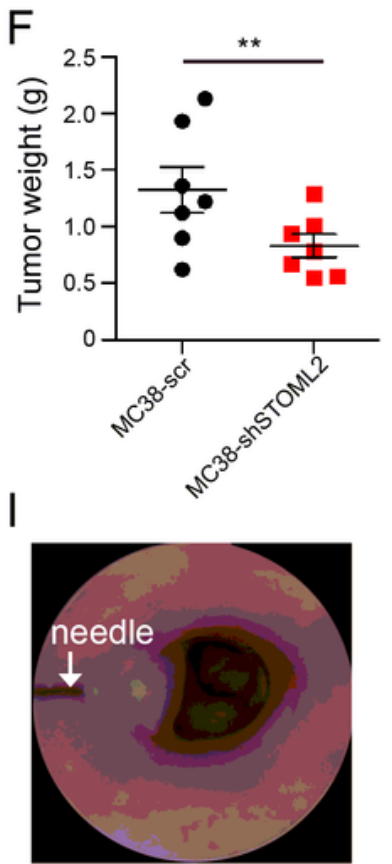

G
B

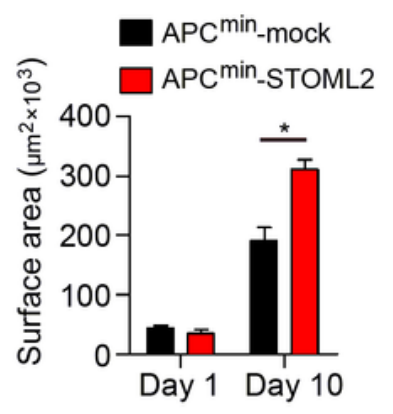

D
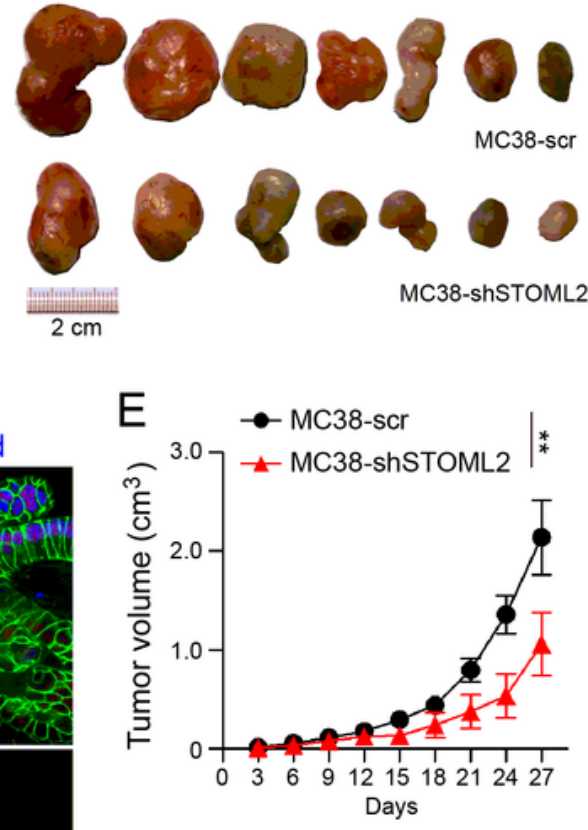

$\mathrm{H}$
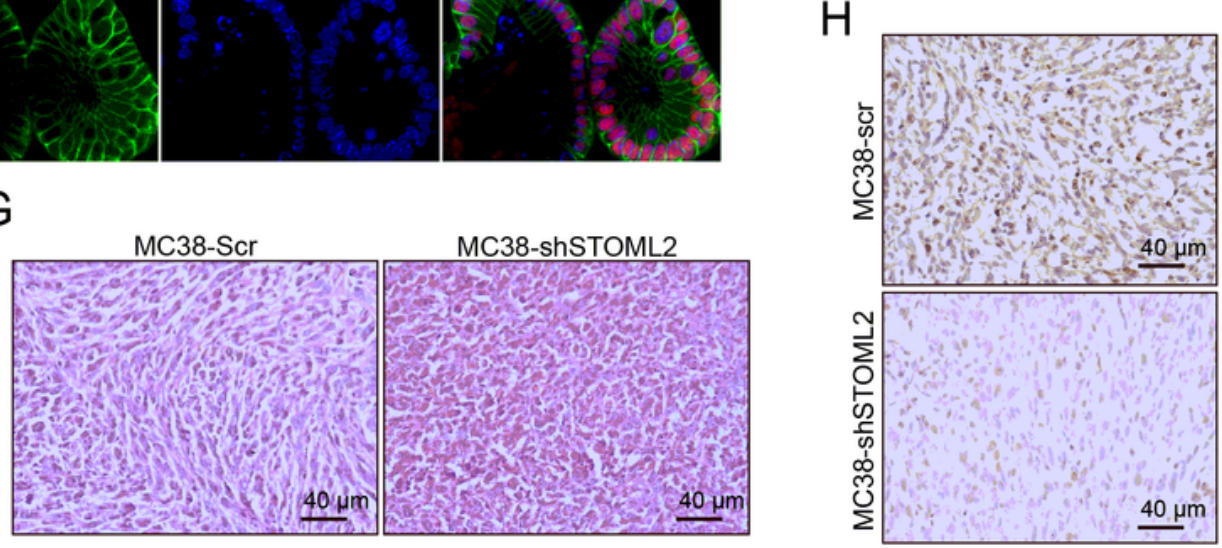

K

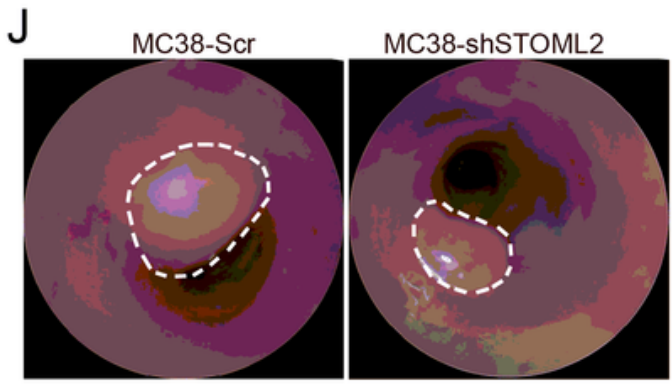

\section{Figure 3}

STOML2 promotes CRC growth and progression in vivo. a-c Organoid culture and subsequent IHC. (a) Representative images of first and tenth day of STOML2-overexpressed organoid culture derived from intestinal tumor of APCmin/+ mice. (b) Calculated surface area ( $\mu \mathrm{m} 2 \times 103)$ of indicated groups of organoid. (c) Immunofluorescent staining to detect Ki67, E-cadherin, DAPI, and merged graphic of indicated organoids (400x). d-h Subcutaneous xenograft of indicated cells in C57B6/J mice. (d) 2×106 
cells were injected into hind limbs of each group, tumors retrieved at 27th day after injection. (e) Tumor volume (cm3) measured at indicated time point. **p囚0.01, two-way ANOVA. (f) Tumor weight (g) measured at indicated time point. ${ }^{*} \mathrm{p} \otimes 0.01, \mathrm{t}$ test. $(\mathrm{g})$ representative image of HE staining of subcutaneous xenograft in each group (400x). (h) representative image of IHC staining to detect Ki67 in subcutaneous xenograft in each group. i-k CRC orthotopic model, murine endoscopy monitoring and survival curve. (i) $1 \times 106$ cells were injected into each $\mathrm{C} 57 \mathrm{~B} 6 / \mathrm{J}$ colon sub-mucosa under endoscopy. White arrow indicates needle. (j) Endoscopy monitoring of orthotopic tumor growth in MC38-Scr and shSTOML2 groups. (k) Kaplan-Meier survival curve of mice in each group. $p$ value was determined using log-rank test. 
A
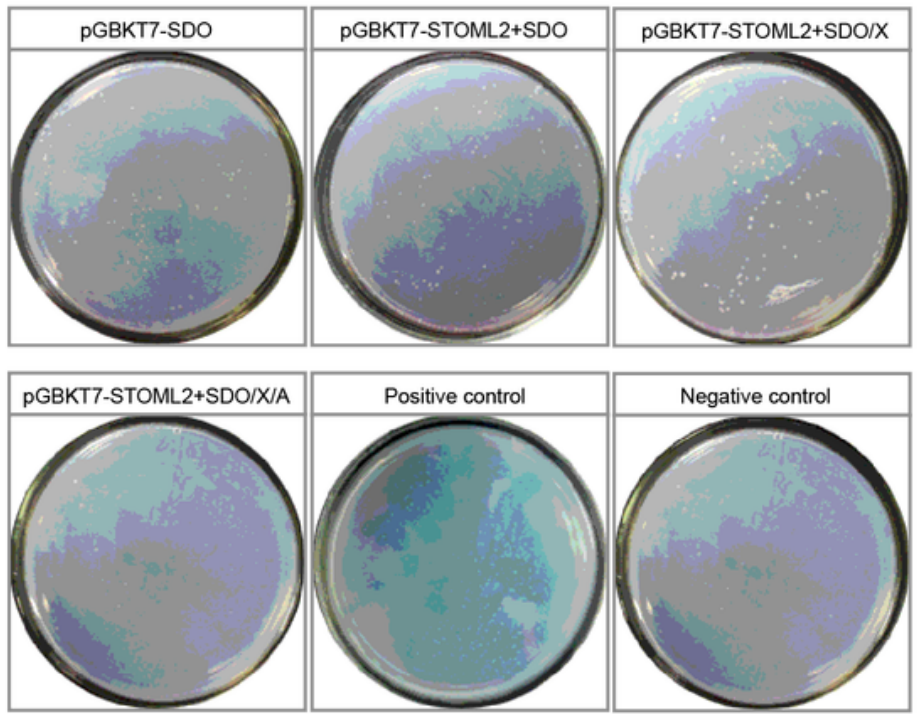

$\mathrm{C}$
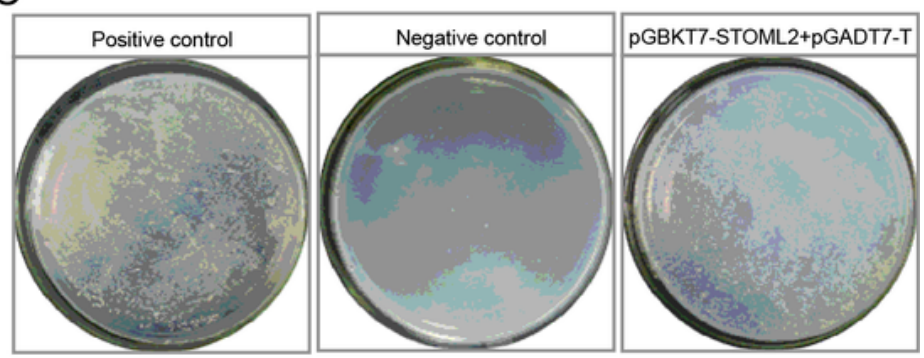

D
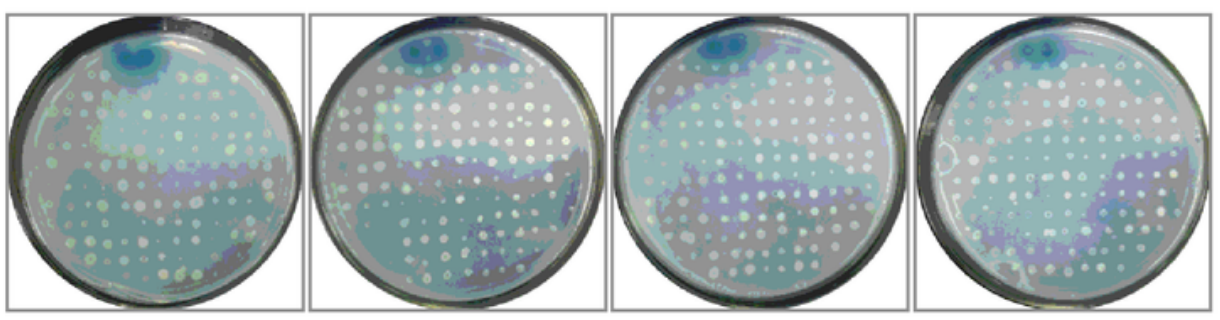

$\mathrm{E}$
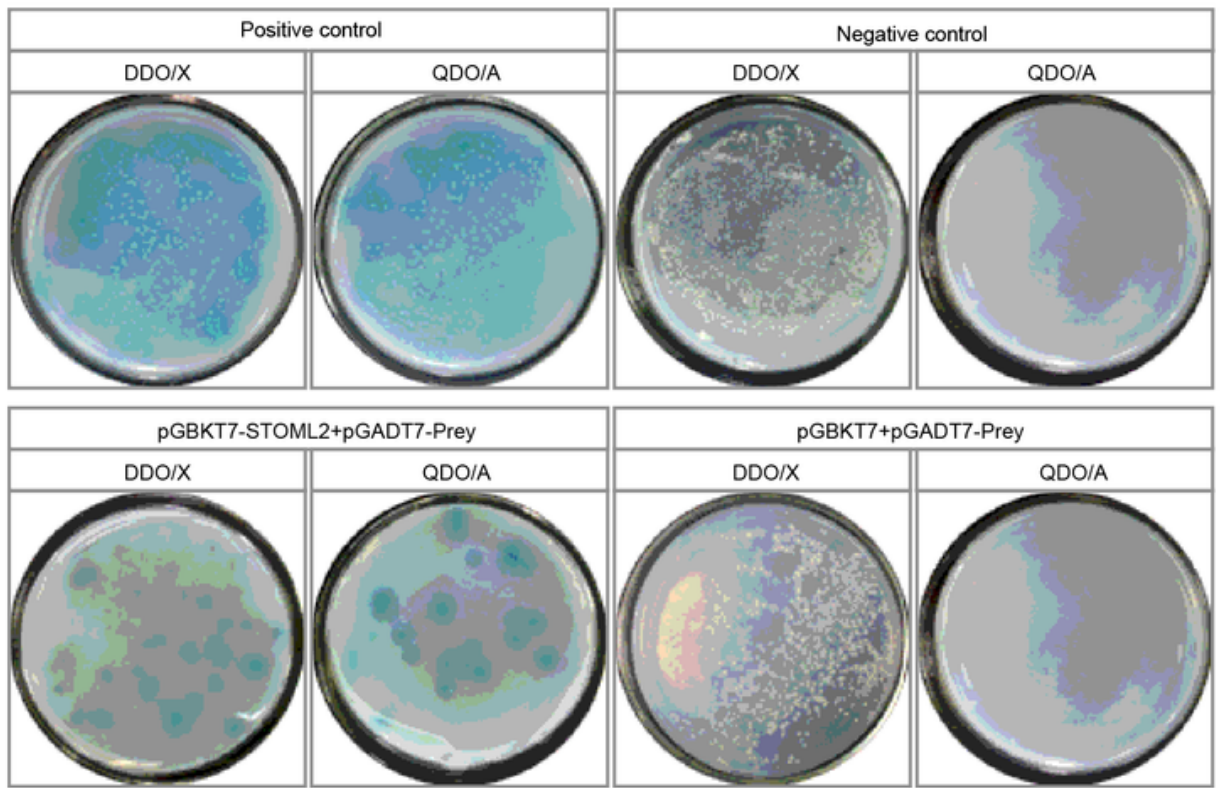

$\mathrm{F}$

Baits: pGBKT7-STOML2 aa29-356 aa 29

$$
\begin{array}{cl}
29 & \\
++++ & \text { pGADT7-FLI1 } \\
++ & \text { pGADT7-PRSS2 } \\
+ & \text { pGADT7-GLP1 } \\
+ & \text { pGADT7-CETN1 } \\
+++ & \text { pGADT7-DTYMK } \\
+++ & \text { pGADT7-GLP1R } \\
++ & \text { pGADT7-ATP5H } \\
+ & \text { pGADT7-ATP5B } \\
+++ & \text { pGADT7-PHB } \\
+++ & \text { pGADT7-PHB2 } \\
+ & \text { pGADT7-TF7L2 } \\
+ & \text { pGADT7-CPVL } \\
+ & \text { pGADT7-CD8a }
\end{array}
$$

\section{Figure 4}

Yeast two-hybrid assay explored potential targets of STOML2. a Bait protein pGBKT7-STOML2 was tested to be non-toxic to host and could not activate reporter gene by itself. Positive control, yeasts cotransfected with plasmids pGBKT7-53 and pGADT7-T; negative control, yeasts co-transfected with pGBKT7-lam and pGADT7-T. Both spread on SD/-Trp/-Leu/X-a-Gal/AbA (DDO/X/A) plate. SDO, single dropout supplements (SD/-Trp). SDO/X (SD/-Trp/X-a-galactosidase). SDO/X/A (SD/-Trp/X-a-gal/Aba). b 
Three-lobe shaped zygotes appeared after mating library strain and bait strain for 20 hours, at 30-50 rpm under $30^{\circ} \mathrm{C}$. c Mating mixture containing pGBKT7-STOML2+pGADT7-T strain was spread on QDO/A (SD/-Ade/-His/-Leu/-Trp/Aba) plate for 3-5 days under $30^{\circ} \mathrm{C}$. Positive and negative control were the same as above. $\mathrm{d}$ Clones $\geq 2 \mathrm{~mm}$ at diameter were transferred to $\mathrm{QDO} / \mathrm{X} / \mathrm{A}$ plates and cultured $\mathrm{at} 30^{\circ} \mathrm{C}$ for days to underwent higher selective pressure. Blue-stained clones indicated possible interaction between STOML2 and target protein. e Prey plasmids in blue-stained clones in d were extracted and sequenced. Plasmids pGBKT7-STOML2+pGADT7-Prey and pGBKT7 +pGADT7-Prey were respectively transfected into yeasts and spread on DDO/X and QDO/A, to validated their interaction. Positive and negative control were the same as above. $f$ Prey plasmids in positive clones were extracted and sequenced. Sequence was looked up in PUBMED-BLAST in search of prey protein. "+" sign indicates higher possibility to interact with STOML2. 
A

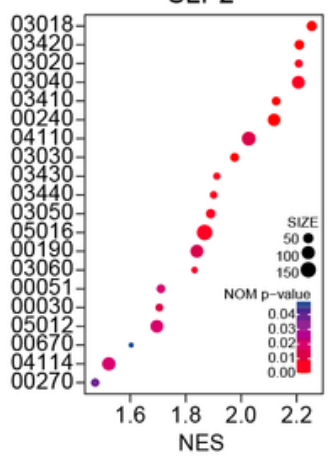

E

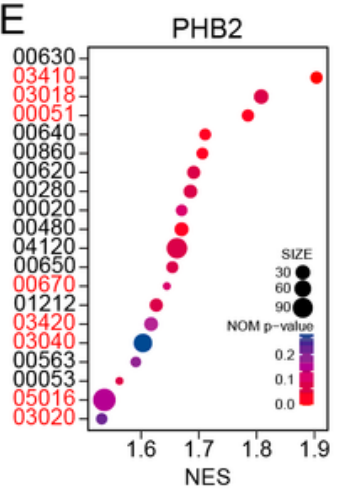

I

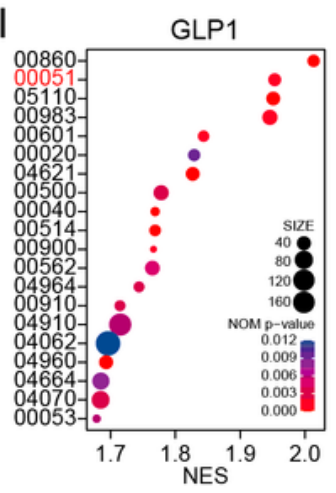

M

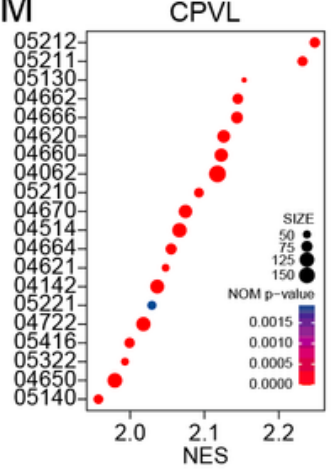

B

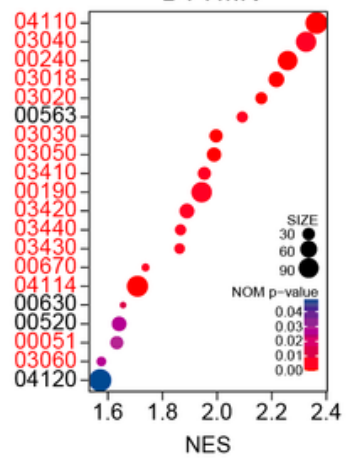

F

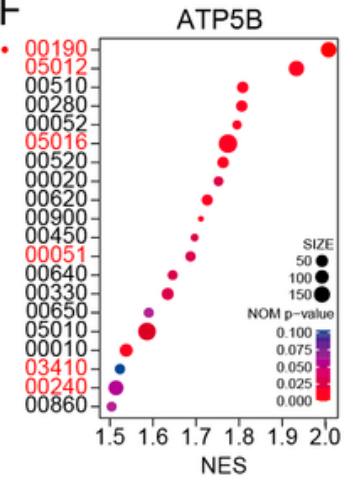

J

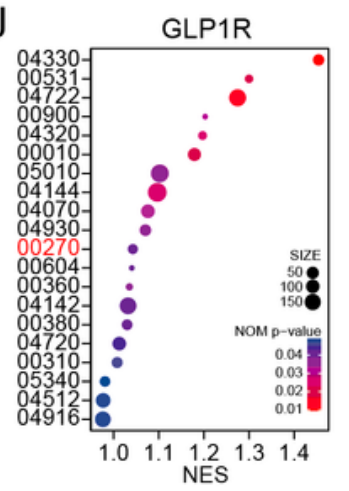

N

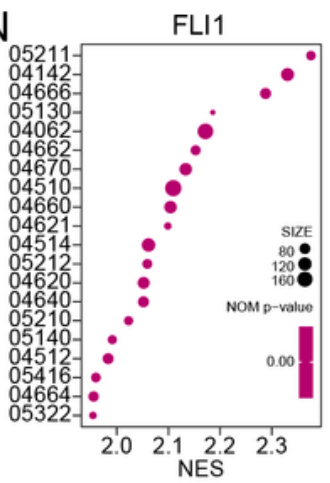

C

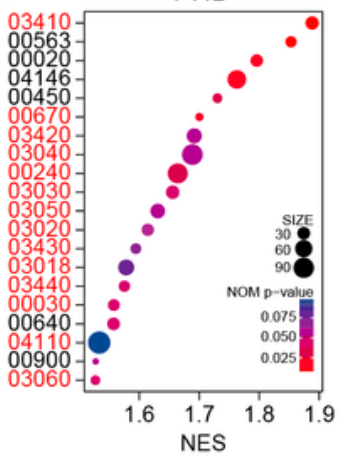

G

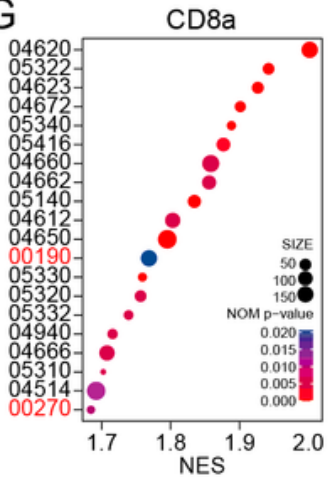

K
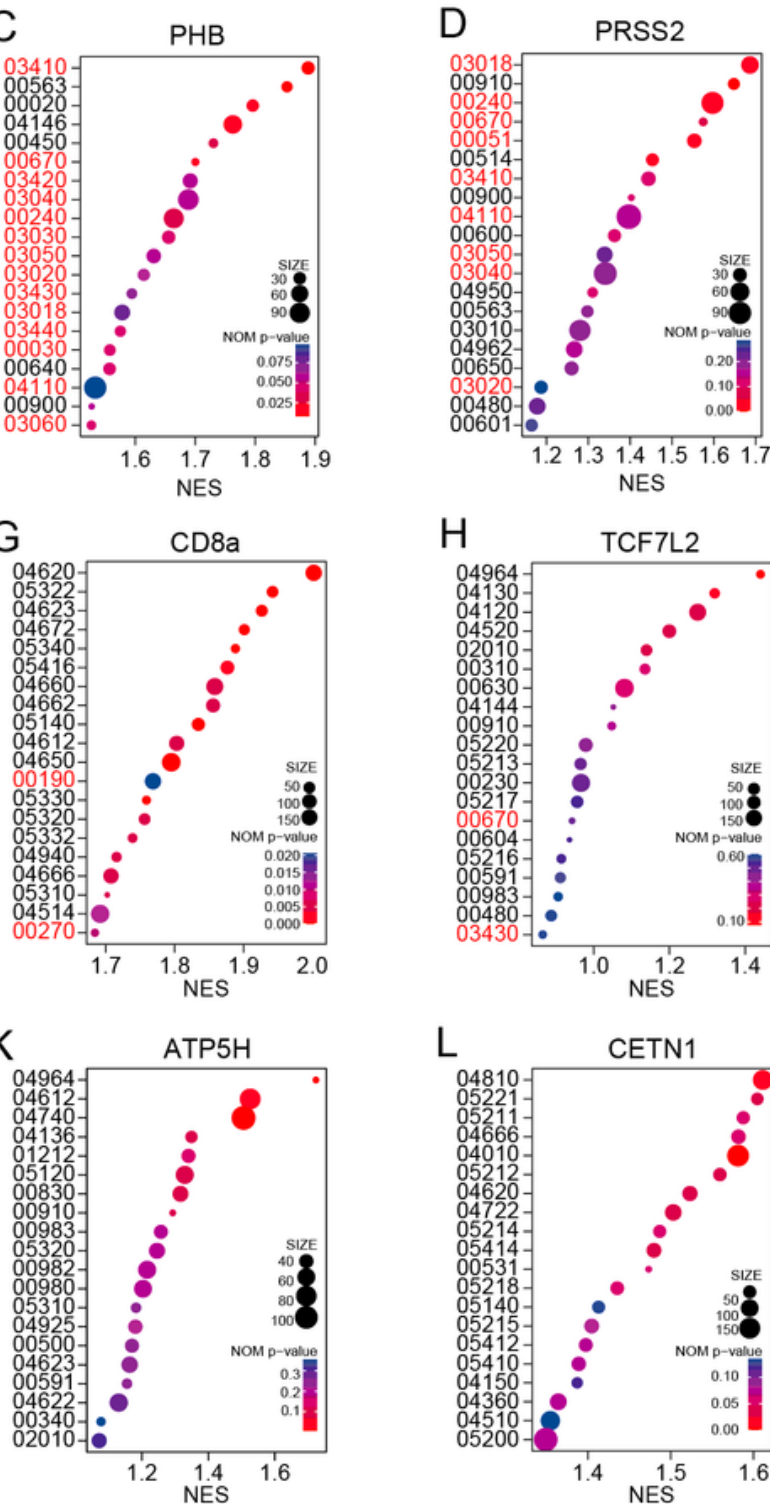

$\mathrm{H}$

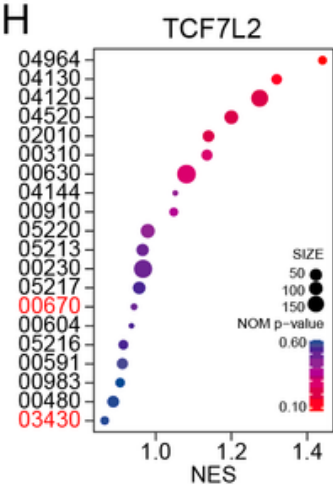

L

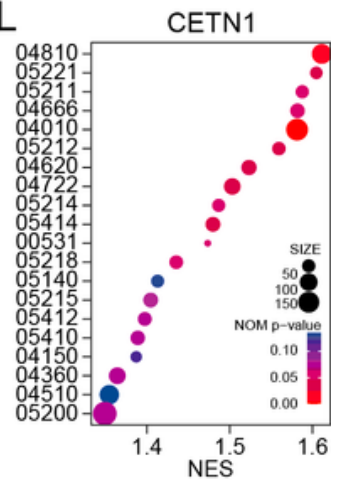

O

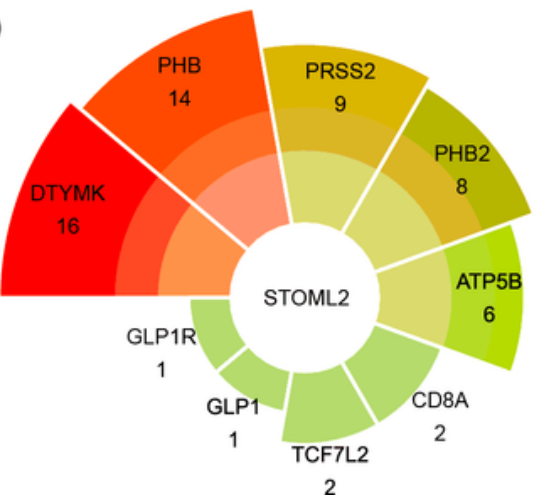

Figure 5

GSEA analyses revealed DTYMK and PHB were functionally similar with STOML2. a-n KEGG pathway of indicated genes in dataset GSE14333. Number on $Y$ axis stands for KEGG pathway terms, red: pathway terms overlapped with STOML2; o statistical diagram of overlapped pathway counts between STOML2 and other genes. 
A

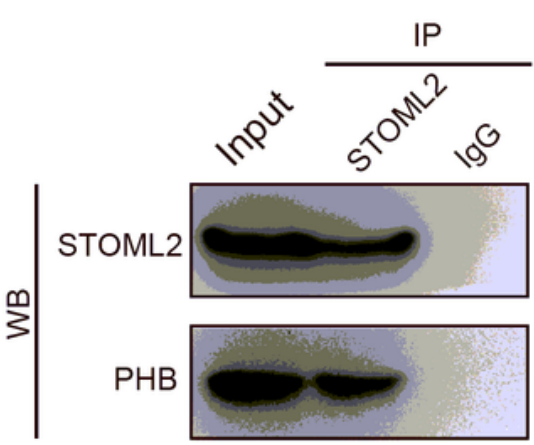

C

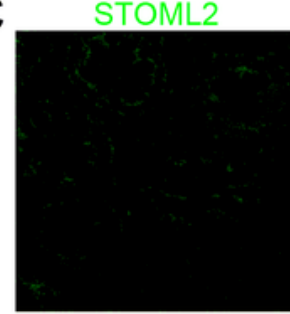

PHB
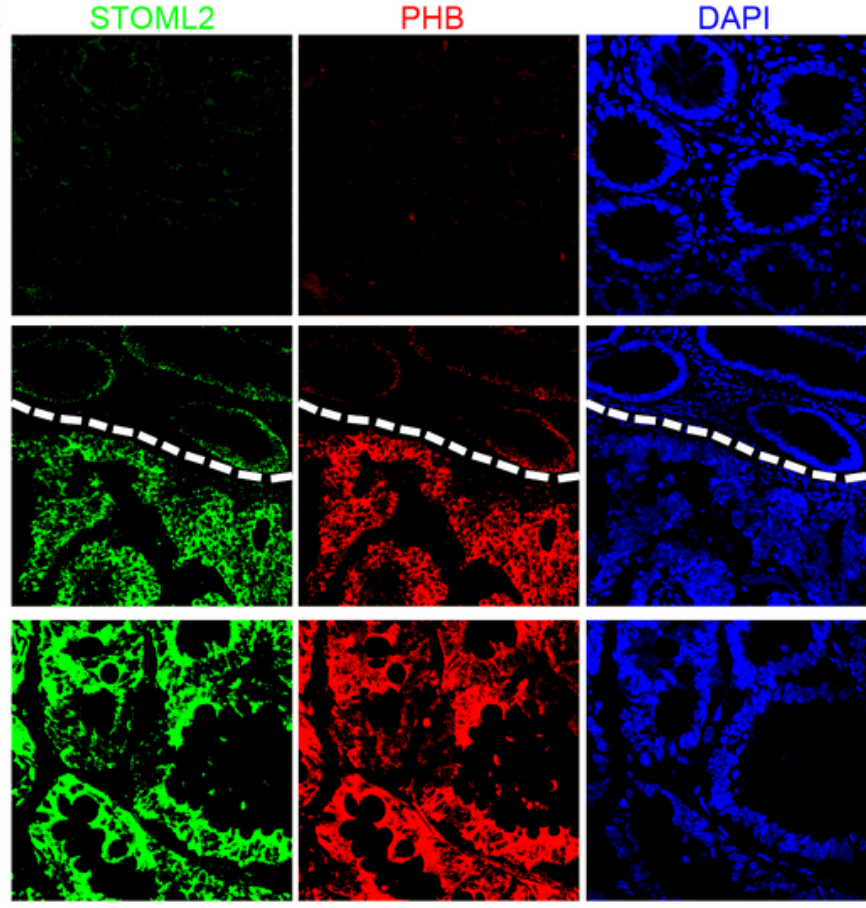

Merged
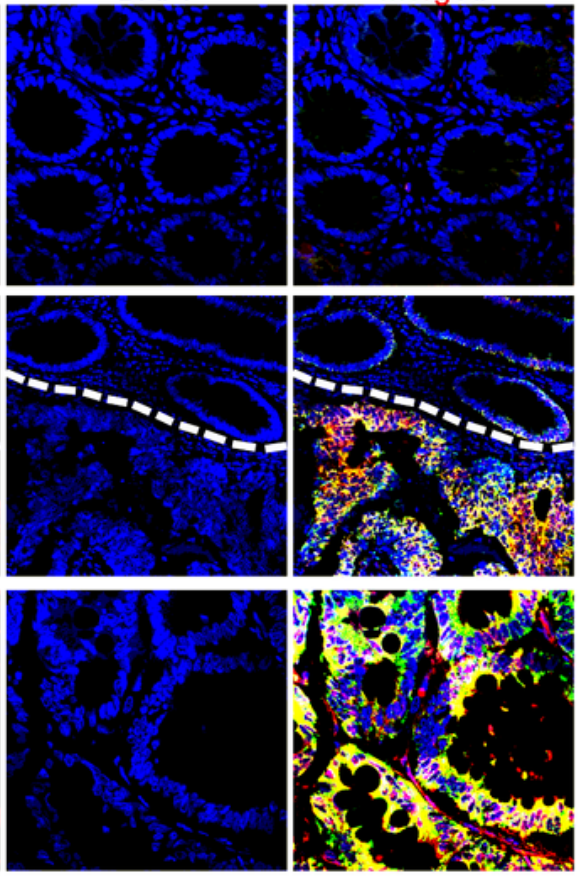

D

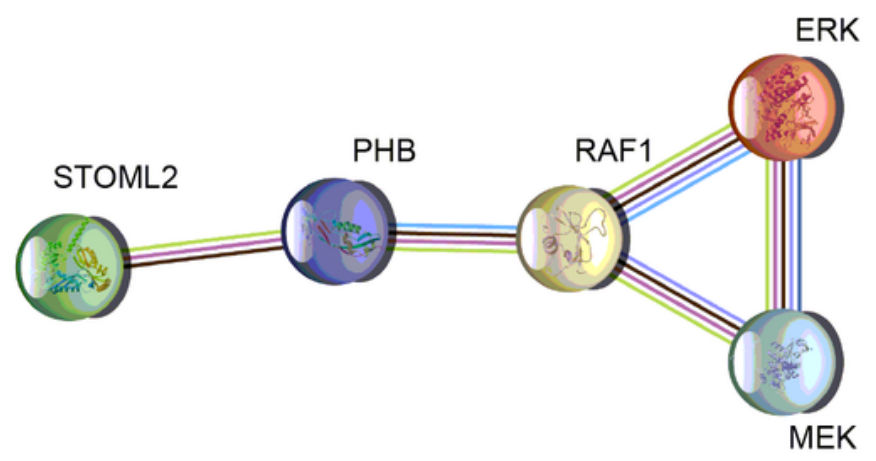

DAPI
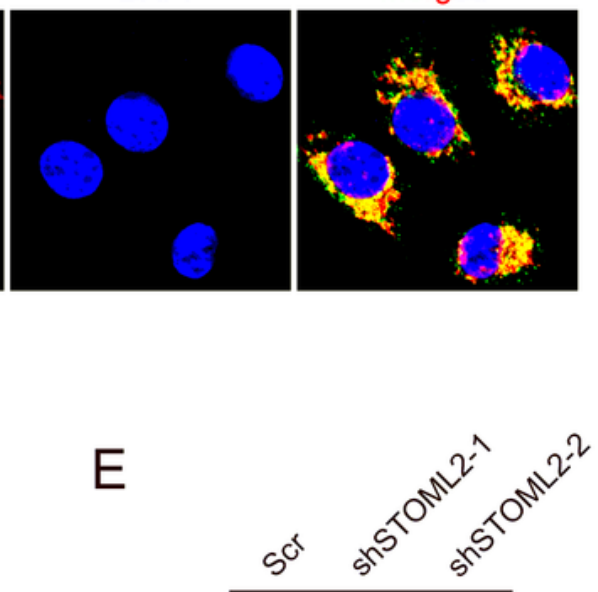

STOML2

PHB
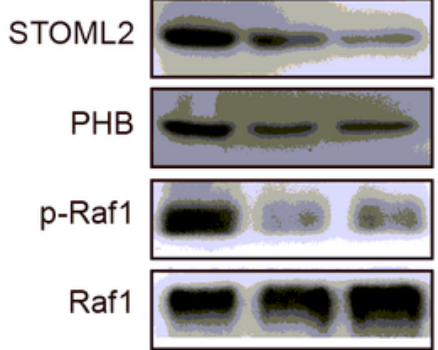

p-MEK1/2

MEK1/2

p-ERK1/2

ERK1/2

GAPDH

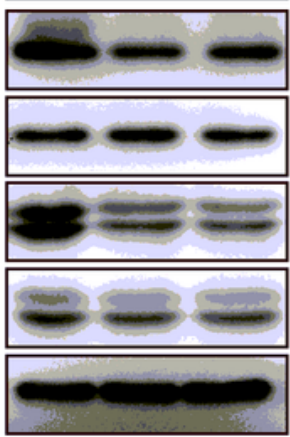

F

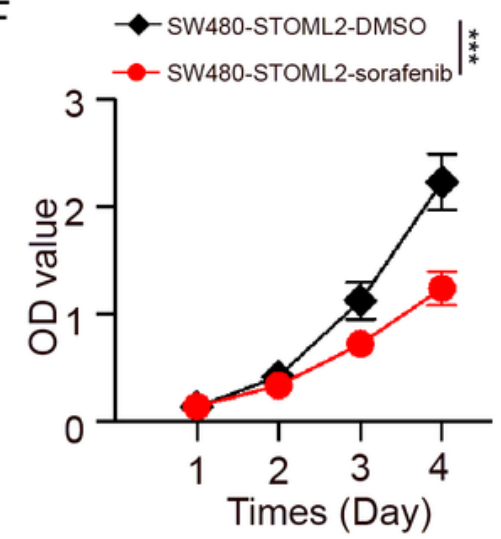

Figure 6

STOML2 interacts with PHB to modulate RAF/MEK/ERK MAPK pathway. a In vitro binding of STOML2 and PHB as shown by Co-IP. b Immunostaining analysis of STOML2 (green), PHB (red) and DAPI for DNA (blue) in SW620. c FFPE sections from CRC tumor adjacent tissue were subjected to immunostaining analysis of STOML2 (green), PHB (red) and DAPI for DNA (blue). d Association of STOML2, PHB, RAF1 and MAPK signaling pathway from STRING database. Interaction score: STOML2 and PHB (0.916); PHB 
and RAF1 (0.965); RAF1 and ERK (0.964); RAF1 and MEK (0.516); MEK and ERK (0.645) e Immunoblots of STOML2, PHB and MAPK signaling pathway from Scr, shSTOML2-1 and shSTOML2-2 transfected SW620 cell. $f$ CCK8 proliferation assay of STOML2-overexpressed SW480 treated with sorafenib or DMSO. Data are presented as means \pm SEM; $* \star \star p<0.001$, two-way ANOVA test.

\section{Supplementary Files}

This is a list of supplementary files associated with this preprint. Click to download.

- SF5.tif

- SF4.tif

- SF3.tif

- SF2.tif

- SF1.tif 